\title{
Comprehensive analysis reveals a metabolic ten-gene signature in hepatocellular carcinoma
}

\author{
Zhipeng Zhu ${ }^{1}$, Lulu Li ${ }^{1}$, Jiuhua Xu ${ }^{2}$, Weipeng Ye ${ }^{2}$, Borong Chen ${ }^{1}$, Junjie Zeng ${ }^{1}$, Zhengjie Huang ${ }^{\text {Corresp. } 1,2}$ \\ ${ }^{1}$ Department of Gastrointestinal Surgery, Xiamen Cancer Center, The First Affiliated Hospital of Xiamen University, Xiamen, Fujian, China \\ 2 Department of clinical medicine, Fujian Medical University, Xiamen, Fujian, China \\ Corresponding Author: Zhengjie Huang \\ Email address: huangzhengjie@xmu.edu.cn
}

Background: Due to the complicated molecular and cellular heterogeneity in hepatocellular carcinoma (HCC), the morbidity and mortality still remains high level in the world. However, the number of novel metabolic biomarkers and prognostic models could be applied to predict the survival of HCC patients is still small. In this study, we constructed a metabolic gene signature by systematically analyzing the data from The Cancer Genome Atlas (TCGA), Gene Expression Omnibus(GEO) and International Cancer Genome Consortium (ICGC). Methods: Differentially expressed genes (DEGs) between tumors and paired non-tumor samples of 50 patients from TCGA dataset were calculated for subsequent analysis. Univariate cox proportional hazard regression and LASSO analysis were performed to construct a gene signature. The Kaplan-Meier analysis, Time-dependent receiver operating characteristic (ROC), Univariate and Multivariate Cox regression analysis, stratification analysis were used to assess the prognostic value of the gene signature. Furthermore, The reliability and validity were validated in four types of testing cohort. Moreover, the diagnostic capability of the gene signature was investigated to further explore the clinical significance, Finally, Go enrichment analysis and Gene Set Enrichment Analysis (GSEA) enrichment analysis have been performed to reveal the different biological processes and signaling pathways which were active in high risk or low risk group. Results: Ten prognostic genes were identified and a gene signature were constructed to predict overall survival (OS). The gene signature has demonstrated an excellent ability for predicting survival prognosis, Univariate and Multivariate analysis revealed the gene signature was an independent prognostic factor. Furthermore, stratification analysis indicated the model was a clinically and statistically significant for all subgroups. Moreover, the gene signature demonstrated a high diagnostic capability in differentiating normal tissue and HCC. Finally, several significant biological processes and pathways have been identified to provide a new insights of the development of HCC. Conclusion: The study have identified ten metabolic prognostic genes and developed a 
prognostic gene signature to provide more powerful prognostic information and improve the survival prediction for HCC . 
1 Comprehensive Analysis Reveals a Metabolic ten-Gene Signature in hepatocellular 2 carcinoma

3

4 5 6

Zhipeng $\mathrm{Zhu}^{1 \dagger}$, Lulu, $\mathrm{Li}^{1 \dagger}$, Jiuhua $\mathrm{Xu}^{2 \dagger}$, Weipeng $\mathrm{Ye}^{2}$, Borong Chen ${ }^{1}$, Junjie Zeng ${ }^{1}$, Zhengjie Huang $1,2^{*}$

${ }^{1}$ Department of Gastrointestinal Surgery, Xiamen Cancer Center, The First Affiliated Hospital of Xiamen University, Xiamen, (Fujian 361003,) P.R. China

${ }^{2}$ Department of clinical medicine, Fujian Medical University, Fuzhou(Fujian 350004), P.R. China

*Corresponding author: Dr. Zhengjie Huang

Address: Department of Gastrointestinal Surgery, Xiamen Cancer Hospital of The First Affiliated Hospital of Xiamen University, 55 Zhen Hai Road, Si Ming District ,Xiamen 361003, Fujian Province, People's Republic of China.

Tel: $+86-592-2139280$

Fax: $+86-592-2137368$

E-mail: huangzhengjie@xmu.edu.cn (Z. Huang)

$†$ Zhipeng Zhu, Lulu, Li, Jiuhua Xu contributed equally to this work 
42 Background: Due to the complicated molecular and cellular heterogeneity in hepatocellular

43 carcinoma (HCC), the morbidity and mortality still remains high level in the world. However, the number of novel metabolic biomarkers and prognostic models could be applied to predict the survival of HCC patients is still small. In this study, we constructed a metabolic gene signature by systematically analyzing the data from The Cancer Genome Atlas (TCGA), Gene Expression Omnibus(GEO) and International Cancer Genome Consortium (ICGC).

Methods: Differentially expressed genes (DEGs) between tumors and paired non-tumor samples of 50 patients from TCGA dataset were calculated for subsequent analysis. Univariate cox proportional hazard regression and LASSO analysis were performed to construct a gene signature. The Kaplan-Meier analysis, Time-dependent receiver operating characteristic (ROC), Univariate and Multivariate Cox regression analysis, stratification analysis were used to assess the prognostic value of the gene signature. Furthermore, The reliability and validity were validated in four types of testing cohort. Moreover, the diagnostic capability of the gene signature was investigated to further explore the clinical significance, Finally, Go enrichment analysis and Gene Set Enrichment Analysis (GSEA) enrichment analysis have been performed to reveal the different biological processes and signaling pathways which were active in high risk or low risk group.

Results: Ten prognostic genes were identified and a gene signature were constructed to predict overall survival (OS). The gene signature has demonstrated an excellent ability for predicting survival prognosis, Univariate and Multivariate analysis revealed the gene signature was an independent prognostic factor. Furthermore, stratification analysis indicated the model was a clinically and statistically significant for all subgroups. Moreover, the gene signature demonstrated a high diagnostic capability in differentiating normal tissue and HCC. Finally, several significant biological processes and pathways have been identified to provide a new insights of the development of HCC.

Conclusion: The study have identified ten metabolic prognostic genes and developed a prognostic gene signature to provide more powerful prognostic information and improve the survival prediction for HCC.

\section{Introduction}

Primary liver cancer was the seventh most commonly occurring cancer in 2018, and the second most common cause of cancer mortality worldwide. The overall 5-year survival of patients with 
83 liver cancer is currently $10-20 \%$. Among them, HCC accounts for most of the primary liver cancer 84 (75\%-85\%), which is characterized by high invasiveness, high metastasis potential and low survival rate.(Bray et al. 2018; Yu et al. 2017) The situation is even more serious in china, liver cancer has a new incidence of 370,000 in 2015, ranking fourth in the number of malignant tumors, 326,000 deaths, and second in the number of deaths.(Zheng et al. 2019) However, it is still lack of effective biomarkers for prediction of high recurrence populations, Death risk and target therapies. Thus, identification of effective biomarker for the prognosis of HCC is urgent for the diagnosis and treatment of HCC.

Traditional serum markers have been proved as potential tumor markers for prognostic in $\mathrm{HCC}$, such as alpha-fetoprotein (AFP).(Hanazaki et al. 2001) However, AFP is only elevated in about half of the HCC patients and significant tumor burden limits its usefulness in screening and operable therapy.(Tangkijvanich et al. 2000) C-reactive protein(CRP) and Platelet lymphocyte ratio(PLR) could be considered as tumor markers for low-AFP HCC patients,(Suner et al. 2019a) and possess parameter values for tumour growth and invasiveness.(Suner et al. 2019b) However, the effects of CRP or PLR on survival reveal unclear. What's more, traditional prognosis markers for HCC only focused on single biomarker, including enzymes and isoenzymes, growth factors and their receptors, tumor-associated antigens, microRNAs (miRNAs) and long noncoding RNAs (lncRNAs),(Mann et al. 2007; Singhal et al. 2012; Yu et al. 2007; Yu et al. 2010) which may lack sensitivity and specificity. With the development of high-throughput technologies, many new potential biomarkers are easier and the gene prognostic signature is more likely to generated for prognosis in $\mathrm{HCC}$.

Altered cellular metabolism plays a key role for cancerous cells, and cancerous cell metabolism reprogramming is considered the novel hallmark of cancer in the future.(Hanahan \& Weinberg 2011) Previous studies have indicated that metabolism alteration could promote cell proliferation and progression, and XR, X. et al(Xu et al. 2001) shown that the transcription level of metabolic genes has changed in HCC. Thus several metabolism-related genes may play a role in the occurrence and development of HCC. However, the number of novel metabolic biomarkers and prognostic models could be applied to predict the survival of HCC patients is still small. Jiang L et al(Jiang et al. 2019) have constructed a glycolysis gene prognostic signature of HCC, but only one validation cohort have been used to prove the performance of the predicted model, and it lacks of comparison of performance with other different biomarker. Rui Benfeitas et al(Benfeitas et al. 2019) built four-gene survival signature of HCC, systematic analysis is required to further prove the predicted value. Inspired by all these works, our research combined with clinically significant metabolic genes to make a gene prognosis model, which could provide better guidance for the survival and prognosis of HCC.

\section{Method}

\section{Identification and acquisition of TCGA and ICGC data}

Gene expression profiles and clinical data associating with $\mathrm{HCC}$ were identified and acquired from TCGA project ( https://cancergenome.nih.gov/ ) , GEO database ( https://www.ncbi.nlm.nih.gov/gds/) and ICGC project ( https://icgc.org/ ). 415 candidates (365 patients and 50 normal candidates) and the clinical information( age, gender, grade, stage, 
124 myometrial invasion, lymph node status, distant metastasis status) were identified and acquired from TCGA dataset. 220 patients and the clinical information (age, gender, ALT, AFP, stage) were identified and acquired from GEO database. 243 patients and the clinical information (gender, age, stage, priorMalignancy) were identified and acquired from ICGC dataset.

Patients characteristics and grouping

365 patients from TCGA dataset, used as the training cohort. Using the R package Caret, with the ratio of $1: 1$ in a random manner, 184 patients from TCGA dataset were selected as the internal testing cohort. 243 patients from ICGC dataset and 220 patients from GSE14520 dataset, used as the external testing cohorts. Finally, we integrated all the 828 patients from the TCGA, GEO and ICGC datasets to use as the entire testing cohort. The clinical information of the four cohorts is summarized in Table 1.

\section{Identification of metabolism related genes}

Metabolism-related genes in the KEGG pathway associated with metabolism were screened from the GSEA (http://software.broadinstitute.org/gsea/index.jsp), and the overlapping metabolismrelated genes were identified from TCGA, GSE14520, and ICGC gene expression profile.

Prognostic genes were identified and gene signature were constructed by utilizing training cohort Using the Limma version 3.36.2 R package, DEGs were calculated between tumors and paired non-tumor samples of 50 patients from TCGA dataset, the adjusted $P$-value $<0.05$ and absolute $\log 2$ fold change $(\mathrm{FC})>1.5$ were considered as the selection criterion. By using the $\mathrm{R}$ package survival and Coxph function, Univariate Cox proportional hazard regression analysis was performed to discover the prognostic genes in the training cohort, with the adjusted $P$-value $<0.05$ as the significance cutoff. We further narrowed the gene range to construct a gene signature by performing LASSO analysis, R package glmnet was used to perform LASSO analysis.

\section{The performance of gene signature}

With the risk-formula: Risk score $=$ expression of gene1 $\mathrm{x} \beta$ 1gene $1+$ expression of gene $2 \mathrm{x}$ $\beta 2$ gene $2+\ldots$. expression of genen $x \beta$ ngenen, patients in training cohort were divided into low or high risk group basing on the median risk score. The expression level was compared between low and high risk group. Kaplan-Meier analysis was performed to compare the survival rate between the two groups. ROC curve analysis for OS was performed to assess the clinically predictive ability of the gene signature. Next, Univariate and Multivariate Cox proportional hazards analysis were performed to investigate whether the gene signature could be independent of other clinical parameters, including age, gender, grade, stage, myometrial invasion, lymph node status, distant metastasis status. Furthermore, stratification analysis were used to assess the prognostic value of the gene signature in different subgroups stratified clinical variables. Moreover, the diagnostic capability of the gene signature was investigated to further explore the clinical significance, including differentiating normal tissue and HCC, different stages and grades. Validation of the gene signature

Internal testing cohort, GSE14520 testing cohort, ICGC testing cohort and entire testing cohort were used to validate the reliability and validity of the gene signature. According to the riskformula and the median risk score, the patients from the four testing cohorts were divided into low or high risk group. And the same analyses were performed to validate the performance, including 
165 Kaplan-Meier analysis, the ROC curve analysis, Univariate and Multivariate Cox proportional 166 hazards analysis, stratification analysis.

167 Go enrichment analysis and Gene set enrichment analysis (GSEA)

168 Metabolism related DEGs were identified between high- and low-risk groups, with corrected P169 value $<0.05$ and absolute log fold change $(\mathrm{FC})>1.5$ being considered as the cutoff criterion. Next, 170 gene ontology processes were considered as enriched by using the DAVID database 171 (https://david.ncifcrf.gov/). Furthermore, we generated an ordered list of all genes according to 172 their correlation with two subtypes and elucidated the significant survival difference between high173 and low-risk groups by GSEA. 1000 times were performed for gene set permutations. The nominal $174 P$ value were used to sort the pathways enriched in each phenotype.

175 Statistical Analysis

$176 P<0.05$ was considered statistically significant, The statistical analyses were conducted by 177 employing the R (version 3.4.3) and GraphPad Prism 7.

178 Result

179 Identification of prognostic genes

180 We carry on our study as described in the flow chart (Figure 1). 944 metabolism-related genes 181 were identified from the online website GSEA (Table S1) and 918 overlapping metabolism-related 182 genes were obtained between TCGA, GSE14520 and ICGC gene expression profile (Table S2). 18371 up-regulated genes and 25 down-regulated genes were calculated for subsequent analysis 184 (Figure 2, Table S3). By conducting Univariate Cox proportional hazards regression analysis, 11 185 significant genes associated with OS were obtained for further analysis (adjusted $P<0.05)($ Table 186 S4).

187 Establishment of gene signature from the training cohort

188 LASSO analysis was performed to narrow the gene range to construct a ten-gene signature from 18911 significant genes, (Risk-formula: Risk score $=$ expression of G6PD x $0.0015558631423743+$ expression of LPCAT1 x 0.00310356967612766 + expression of ME1 x $0.00632401235412166+$ expression of PRIM1 x $0.0026285256475841+$ expression of RRM2 x $0.00979851158278189+$ expression of TXNRD1 x $0.00783495109084+$ expression of UCK2 x $0.057281553558209+$ expression of CAD x 0.06164749118803 + expression of DTYMK x $0.00849922964649245+$ expression of ENTPD2 $\times 0.03691738437542)$. The risk score was computed for each patient in the training cohort, 365 patients from training cohort were divided into low risk group (183 patients) and high risk group (182 patients) according to the median risk score: 0.73. 243 patients from ICGC testing cohort were divided into low risk group(100 patients) and high risk group (143 patients). 220 patients in GSE14520 testing cohort were divided into low risk group(112 patients) and high group(108 patients). 184 patients in the internal testing cohort were divided into low risk group ( 95 patients) and high risk group ( 89 patients), 828 patients in the entire testing cohort were divided into low risk group(356 patients) and high risk group (432 patients). The ten prognostic genes expression level distribution between low and high risk group of the training cohort was showed in Figure 3A, the expression of ten prognostic genes in high risk group was higher than in low risk group, the result was consisted with internal testing cohort in Figure 3B, GSE14520 testing cohort in Figure 3C and ICGC testing cohort in Figure 3D. 
206

207

208

209

210

211

212

213

214

215

216

217

218

219

220

221

222

223

224

225

226

227

228

229

230

231

232

233

234

235

236

237

238

239

240

241

242

243

244

245

246

The performance of gene signature

As showed in Figure 4A, with the increasing risk score, patients in the training cohort have a worse OS, the expression ten prognostic genes increased. The ROC curve was presented to assess the clinically predictive ability of the gene signature, the AUC for 1-year(Figure 4B), 3-year(Figure 4C), and 5-year(Figure 4D) OS were $0.805,0.756,0.716$ for training cohort, which was higher than other clinical characteristics, including age $(0.512,0.536,0.540)$, gender $(0.504,0.535,0.565)$, grade $(0.522,0.505,0.527)$, stage $(0.659,0.688,0.669)$. Besides, we found the gene signature also performed more specific and sensitive than any single gene (Table 2). The OS in the training cohort was significantly different between low and high risk group, in the 1-year, 3-year and 5-year, the OS in the high risk group were $0.576,0.181,0.076$, the OS in the low risk group were 0.814 , $0.306,0.142$, the result indicated that patients with a high-risk score have more poor OS than the patients with low-risk $\operatorname{score}(P<0.001)$, detail was presented in Figure 4E. By conducting Univariate and Multivariate Cox regression analysis, we noted that gene signature have a significant correlation with worse OS, the HR of the gene signature was $3.919(95 \% \mathrm{CI}=2.766$ 5.555) with $P$-value $<0.001$ in Univariate Cox regression analysis (Figure 4F), 3.490 (95\% $\mathrm{CI}=2.401-5.073$ ) with $P$-value $<0.001$ in Multivariate Cox regression analysis (Figure $4 \mathrm{G}$ ), thus the gene signature was an independent prognostic factor of other clinical variables.

Validation of the gene signature

To validate the predictive ability in different HCC populations, we applied the gene signature to ICGC testing cohort, the result was similar to the training cohort. Figure 5A showed the distribution of risk scores for each patients, patients in high risk group had a worse OS than patients with a low-risk group. In addition, the AUC for 1-year(Figure 5B), 3-year(Figure 5C), and 5year(Figure 5D) OS were $0.775,0.754,0.778$, which was higher than other clinical characteristics, including age $(0.542,0.520,0.595)$, gender $(0.587,0.574,0.545)$, priorMaliganancy $(0.526,0.572$, 0.508). Even though the AUC of gene signature was a little less than the TNM stage at 1-year $\mathrm{OS}(0.775$ vs 0.809$)$, the AUC of gene signature was much larger than the TNM stage at 3-year OS(0.754 vs 0.658$), 5$-year OS(0.778vs0.564). The AUC of ROC for gene signature was obviously greater than single gene (Table 2). Moreover, the OS for patients in the high risk group was 0.576 at 1-year, 0.181 at 3-year, 0.076 at 5-year, compared with $0.814,0.306,0.142$ in the low risk group $(P<0.001$, Figure 5E). Further Univariate Cox regression analysis and Multivariate Cox regression analysis displayed gene signature was a powerful and independent factor in external testing cohort (Figure 5F, 5G). For GSE14520 testing cohort(Figure 6), internal testing cohort(Figure 7) and entire testing cohort(Figure 8), the gene signature had the similar predictive ability. The distribution of risk scores, gene expression were evaluated in GSE14520 testing cohort(Figure 6A), internal testing cohort(Figure 7A) and entire testing cohort(Figure 8A). The ROC curve demonstrated that gene signature was more specific and sensitive than any clinical characteristics and any single gene in GSE14520 testing cohort(Figure 6B,C,D, Table 2), internal testing cohort(Figure 7B,C,D,Table 2) and entire testing cohort(Figure 8 B,C,D, Table 2). Patients in a high-risk group have poorer OS than the patients in low-risk group for GSE14520 testing cohort(Figure 6E), internal testing cohort(Figure 7E), entire testing cohort(Figure $8 \mathrm{E})(P<0.001)$. Univariate and Multivariate Cox regression analysis indicated the gene signature was an 
247 independent prognostic factor for GSE14520 testing cohort(Figure 6F,G), internal testing

248

249

250

251

252

253

254

255

256

257

258

259

260

261

262

263

264

265

266

267

268

269

270

271

272

273

274

275

276

277

278

279

280

281

282

283

284

285

286

287

cohort(Figure 7F,G) and entire testing cohort(Figure 8F,G).

\section{Stratification analysis}

To further demonstrate the clinical significance of the gene signature in HCC, we perform the survival analysis stratified by clinical variables (age, gender, grade, stage) in training cohort and internal testing cohort, by clinical covariates (age, gender, stage) in ICGC testing cohort, GSE14520 testing cohort and entire testing cohort. Patients of stage I-II, stage III-IV, grade1-2, grade $3-4$, age $<60$, age $>=60$, female, male were stratified into high risk group and low risk group. The log-rank test indicated that HCC patients in high risk group still had obviously worse OS than patients in low risk group for training cohort(Figure 9A), internal testing cohort (Figure 9B), ICGC testing cohort (Figure 9C), GSE14520 testing cohort(Figure 9D) and entire testing cohort (Figure 9E), and the high-risk patients of subgroup subdivided by the signature had poorer survival than the low-risk patients in training cohort (Figure S1 A-H), internal testing cohort (Figure S1 I-P), GSE14520 testing cohort (Figure S2 A,B,C,E,F), ICGC testing cohort(Figure S2G-L) and entire testing cohort ( FigureS3A-F). There was no different trend between high and low risk group for female patients in GSE14520 testing cohort, small number female patients may be an important reason. Interestingly, subgroup stage I, stage II, stage III in entire testing cohort were stratified into high risk group and low risk group, HCC patients in high risk group had poorer OS than patients in low risk group (FigureS3G-I).

Bidkhori et.al(Bidkhori et al. 2018) published a paper that was proposing 3 sub-types of HCC, including $\mathrm{iHCC} 1, \mathrm{iHCC} 2$ and $\mathrm{iHCC} 3$. They reported that the expression of iHCC3 tumors are markedly distinct from those of $\mathrm{iHCC} 2$ and $\mathrm{iHCC} 1$, and a larger number of genes are differentially expressed between $\mathrm{iHCC} 3$ and $\mathrm{iHCC} 1 / \mathrm{iHCC} 2$ compared with $\mathrm{iHCC} 1$ versus $\mathrm{iHCC} 2$. Consistent with the result, we revealed that the expression level of majority of genes of the gene signature were higher in $\mathrm{iHCC} 3$ than $\mathrm{iHCC} 1 / \mathrm{iHCC} 2$, and the expression level of iHCC1 was similar to iHCC2(Figure S4).

\section{Diagnostic capability}

The gene signature have been validated the predictive ability in different HCC populations. To further explore diagnostic capability of the gene signature, we compared risk score between normal liver and HCC in training cohort(Figure S5A, $P<0.0001$ ), ICGC testing cohort(Figure S5B, $P<0.0001$ ) and GSE14520 testing cohort(Figure S5C, $P<0.0001$ ), the risk score in HCC was obviously higher in than normal liver. The AUC of ROC curve was 0.98 in training cohort(Figure S6A), 0.77 in ICGC testing cohort(Figure S6B) and 0.97 in GSE14520 testing cohort(Figure S6C), which revealed the strong diagnostic capability for HCC. In addition, we also investigated the distribution of high-risk and low-risk patients in different stages and grades, the proportion of high risk patients is higher in advanced tumor grade ( grade 3-4) than early tumor grade (grade 1-2) in training cohort (Figure S5D). The proportion of high risk patients is higher in late stage (TNM IIIIV) than early stage (TNM I-II) in training cohort (Figure S5E), ICGC testing cohort(Figure S5F) and GSE14520 testing cohort (Figure S5G). Furthermore, as the TNM stage and tumor grade increased, the risk score increased (Figure S5H,I,J,K). For early and advanced tumor grade in training cohort( Figure S6D,E), early and advanced TNM stage in training cohort(Figure S6F,G),

Peer) reviewing PDF | (2020:01:45125:1:1:NEW 6 Apr 2020) 
288 ICGC testing cohort(Figure S6H,I), GSE14520 testing cohort(Figure S6J,K), the AUC of ROC 289 curve indicated the modest diagnostic capability.

290 Comparing the performance of the gene signature with other gene signatures and TMN stage

291 TMN stage is still significant to predict the survival of HCC patients. In the training cohort, we 292 found the AUC of gene signature was larger than the TNM stage at 1-year, 3-year, 5-year(0.805 293 vs 0.659 at 1 -year, 0.756 vs 0.688 at 3 -year, 0.716 vs 0.669 at 5 -year ) (Figure 4 B,C,D), the result 294 was consistent with GSE14520 testing cohort(Figure 6B,C,D), internal testing cohort (Figure 295 7B,C,D) and entire testing cohort( Figure 8B,C,D). For ICGC testing cohort(Figure 5B,C,D), even 296 though the AUC of gene signature was a little less than the TNM stage at 1-year OS(0.775 vs 297 0.809), the AUC of gene signature was much larger than the TNM stage at 3-year OS(0.754 vs 298 0.658),5-year OS(0.778 vs 0.564). Thus we believed the gene signature was more specific and 299 sensitive than TNM stage. Next, we also compared the AUC of ROC between the gene signature 300 and all single genes, the AUC of the gene signature was larger than any single gene (Table 2). 301 Moreover, we further compared the gene signature with other gene signatures, the ROC analysis 302 indicated our model had better performance, 0.805 at 1-year, 0.756 at 3-year, 0.716 at 5-year, the 303 AUC of Long et al's (Long et al. 2018) model is 0. 7674, 0.7040, 0.6919 at 1, 3 and 5-year, the 304 AUC of Qiao et al's(Qiao et al. 2019) model is 0.71, 0.69 at 3 and 5-year, the AUC of Li et al et 305 al's(Li et al. 2017) model is 0.67, 0.67 at 3 and 5-year, the AUC of Xiao-Hong Xiang al's(Xiang 306 et al. 2019) model is $0.708,0.699,0.678$ at 1, 3 and 5-year, the AUC of Li et al's(Li et al. 2017) 307 model is 0.727, 0.709, 0.604 at 1,3 and 5-year, the AUC of Yan et al's(Yan et al. 2019) model is $3080.712,0.661$ at 1, 3 -year, indicating that the model had a high sensitivity and specificity for predict 309 the survival.

310 Go enrichment analysis and Gene set enrichment analysis (GSEA)

311 We performed a differential expression analysis between low and high risk group to reveal the 312 association between the metabolic genes and two subtypes. We identified 18 upregulated and 14 313 downregulated genes between patients in the low vs high risk group. Go enrichment analysis 314 revealed that upregulated genes in high risk group were mainly involved in cell cycle regulation, 315 such as DNA replication, chromatid/chromosome segregation and regulation, spindle organization 316 and recombination. Upregulated genes in low risk group were mainly involved metabolic and 317 energy regulation, including metabolism of amino acids, oxidative phosphorylation, fatty acid $\beta$ 318 oxidation and catabolism(Figure S7A).

319 In order to further explore the mechanism of prognostic genes in patients with hepatocellular 320 carcinoma, we conducted GSEA between low and high risk group to identify the significant 321 pathways $(\mathrm{FDR}<0.05$, NOM $P$-value $<0.05)$. The most meaningful pathway were identified according to the FDR standard, we uncovered the most meaning pathways which were active in the high-risk group, including KEGG_CELL_CYCLE, KEGG_NUCLEOTIDE_EXCISION_REPAIR, KEGG_OOCYTE_MEIOSIS KEGG_PURINE_METABOLISM, KEGG_PYRIMIDINE_METABOLISM. And the most meaning pathways which were active in the low-risk group, including KEGG_DRUG_METABOLISM_CYTOCHROME_P450,KEGG_FATTY_ACID_METABOLI 328 SM,KEGG_GLYCINE_SERINE_AND_THREONINE_METABOLISM,KEGG_PRIMARY_BI 
LE_ACID_BIOSYNTHESIS,KEGG_VALINE_LEUCINE_AND_ISOLEUCINE_DEGRADAT ION. The result was consistent with go enrichment analysis, significant pathways which were active in high risk group were mainly related with cell cycle regulation. In contrast, meaningful pathways which were active in low risk group were mainly associated with metabolic and energy regulation (Figure S7B).

\section{Discussion}

Due to the complicated molecular and cellular heterogeneity in HCC, the morbidity and mortality still remains high level in the world. Novel prognostic biomarkers to predict the survival of HCC patients is urgently needed. Metabolism reprogramming is considered the novel hallmark of cancer in the future. However, the number of novel metabolic biomarkers and prognostic models could be applied to predict the survival of HCC patients is still small. In this study, we constructed a metabolic gene signature by performing training cohort from TCGA dataset, the gene signature showed a strong prognostic performance for predicting the survival of HCC patients. Compared with previous studies and TNM stage, the model possessed a higher sensitivity and specificity. Meantime, the gene signature was an independent prognostic factor of other clinical variables, which showed a high value of HR. The prognostic value of the gene signature was validated by performing internal testing cohort, ICGC testing cohort, GSE14520 testing cohort and entire testing cohort. Furthermore, the gene signature demonstrated a high diagnostic capability in differentiating normal tissue and HCC, and showed a modest diagnostic capability in early and advanced TNM stage, early and advanced grade. Finally, several significant biological processes signaling pathways underlying hepatocellular carcinoma were identified for further validation.

We identified ten risky prognostic genes (CAD, DTYMK, ENTPD2, G6PD, ME1, RRM2, TXNRD1, UCK2, LPCAT1, PRIM1). CDA gene encodes a 243-kDa multifunctional protein, consists of carbamyl phosphate synthetase (CPSase), aspartate transcarbamylase (ATCase), glutamine amidotransferase (GLNase), dihydroorotase (DHOase).(Kim et al. 1992) CDA is the main participant in de novo pyrimidine synthesis, which is very important to provide malignant cells and proliferating cells with nucleotides for DNA replication.(Aoki \& Weber 1981; Fairbanks et al. 1995) Therefore, the upregulation of $C A D$ may be considered as a prognosis biomarker and therapeutic target. Uhlén $\mathrm{M}$ et al(Uhlen et al. 2015) have reported that the expression level of $C A D$ is high, Morin A et al(Morin et al. 2012) indicated the expression level of $C A D$ was associated with local tumor extension and cancer relapse and identified $C A D$ as a potential predictive marker of cancer relapse, Sigoillot FD et al(Sigoillot et al. 2004) have showed the intracellular $C A D$ concentration was 3.5- to 4-fold higher in MCF7 cells than that in normal MCF10A breast cells, and MAP kinase activity and a nonclassical ERalpha/Sp1-mediated pathway may account for the high $C A D$ level.(Khan et al. 2003) Previous study have indicated the expression level of $C A D$ is higher in hepatoma carcinoma cell than normal liver cell, however, The prognostic value of $C A D$ for hepatocellular carcinoma has not been validated. DTYMK is a nuclear-encoded deoxythymidylate kinase, which expressed in all tissues and participate in the activity of dTTP production,(Caspi et al. 2016) and is a key part for DNA synthesis. Yan Liu et al(Liu et al. 2013b) have indicated the expression of DTYMK is increased in lung adenocarcinomas in comparison to normal lung, and identified elevated DTYMK expression as an unfavorable predictor. Hsi-Wen 
370 Yeh et al(Yeh et al. 2017) found the DTYMK was a poor prognostic factor in HCC. DTYMK was 371 observed in the 5-FU resistant colon cancer cells, which may provide a new therapy for the HCC 372 by applying the 5-FU combination therapy. ENTPD2 belongs to ENTPD family, Stagg J et al(Chiu 373 et al. 2017) have reported only ENTPD1 plays an important role in cancer, however, discloses that 374 ENTPD2 is harnessed by cancer cells to escape immune-mediated destruction and the expression 375 level of ENTPD2 was also high in HCC patients, and the high expression of ENTPD2 was 376 associated with direct liver invasion, tumor microsatellite formation and venous invasion, as well 377 as the absence of tumor encapsulation. The pentose phosphate pathway belongs to major 378 carbohydrate pathways, it could produce ribose and NADPH to protect and promote cells proliferation in hypoxic conditions, such characteristic meets the demand of malignant proliferating cells, therefor, the change of the pentose phosphate pathway may be the landmark of cancer.(Sacoman et al. 2012) G6PD is the rate-controlling enzyme of pentose phosphate pathway, previous researches have reported $G 6 P D$ gene is an oncogene and the expression level upregulates in bladder cancer,(Ohl et al. 2006) ESCC, (Wang et al. 2016)breast cancer,(Pu et al. 2015) Sun Y et al(Sun et al. 2014) indicated G6PD-deficient women have reduced breast cancer risk, Zhang X et al indicated overexpression of G6PD increases the risk of colon cancer, Wang $\mathrm{J}$ et al(Wang et al. 2012a) reported $G 6 P D$ could promote the progression of gastric cancer cells and is associated with poor clinical outcome for patients with gastric cancer.(Zhang et al. 2017) Masayoshi Munemoto et al(Munemoto et al. 2019) activated G6PD gene to accelerates carcinogenesis and cancer progression. Previous studies have detected the G6PD is overexpressed in HCC,(Li et al. 2012; Xu et al. 2014) and Yanyan Gao et al(Gao et al. 2017) found G6PD WAS significantly changed by using sequential window acquisition of all theoretical mass spectra (SWATH-MS), Hu $\mathrm{H}$ et al found the cell migration and invasion ability decreased when the expression of $G 6 P D$ was downregulated. miR122 and miR-1 suppress the expression of G6PD to inhibit tumor growth through inhibiting the activity of PPP in hepatocellular cancer.(Barajas et al. 2018) Lu M et al(Zhao et al. 2018) found G6PD promotes migration and invasion of hepatocellular carcinoma cells through inducing epithelial-mesenchymal transition by activating of transcription 3 (STAT3) pathway. ME1 is multifunctional protein, which relates glycolytic and citric acid cycles. ME1 plays an important role in tumor development, it have reported that the expression level of $M E 1$ is high in various cancers and promotes growth and metastasis, including colorectal cancer, 39 breast cancer,(Liao et al. 2018) bladder cancer,(Liu et al. 2018) gastric cancer,(Lu et al. 2018) nasopharyngeal carcinoma.(Zheng et al. 2012) Several studies indicates that ME1 is associated with poor prognosis in hepatocellular carcinomas and OSCC,(Knoblich et al. 2014; Wen et al. 2015)ME1 promotes HCC metastasis through influencing epithelial-mesenchymal transition (EMT) processes,(Wen et al. 2015) and ME1 also could reduce the sensibility of radiation.(Chakrabarti 2015; Woo et al. 2016) As a vital subunit of rate-limiting catalyzes (ribonucleotide reductase) (RNR), which is necessary for DNA replication and DNA damage repair.(Aye et al. 2015) $R R M 2$ was reported to be associated with various cancers, including ovarian cancer,(Wang et al. 2012b) bladder cancer,(Morikawa et al. 2010b) colorectal cancers,(Liu et al. 2013a; Lu et al. 2012) gastric cancer.(Morikawa et al. 2010a)Meantime, several showed upregulated $R R M 2$ promotes tumorigenesis, proliferation, and inhibits apoptosis, and is associated 
411 with poor prognosis.(Das et al. 2019; Kolberg et al. 2017; Liang et al. 2019; Liu et al. 2013a; 412 Souglakos et al. 2008; Wang et al. 2012b)It is known that RRM2 promotes drug resistance in 413 various cnacers,(Goan et al. 1999; Nakano et al. 2007; Shah et al. 2014) Thus RRM2 is consider a 414 novel drug target,(Aye et al. 2015; Minami et al. 2015) including Trans-4,4'415 Dihydroxystilbene,(Chen et al. 2019)COH29,(Chen et al. 2015)GW8510.(Hsieh et al. 2016)For 416 hepatocellular carcinoma, many bioinformatics analysis indicated $R R M 2$ is a clinical prognostic 417 markers,(Dawany et al. 2011; He et al. 2017; Wu et al. 2019) Boin Lee et al showed the expression 418 level of $R R M 2$ is up-regulated and $R R M 2$ is a significant marker for predicting clinical 419 prognosis.(Lee et al. 2014) Several drug may target $R R M 2$ to suppress hepatocellular carcinoma 420 cells.(Gao et al. 2013; Kosakowska-Cholody et al. 2009) Cancerous cells have to face the increased oxidative stress due to the high metabolism and metabolic disorders, the activation of glutathione (GSH) and thioredoxin (TXN) systems could compensate the severe stress,(Arner \& Holmgren 2006) thus cancerous cells overactivated GSH and TXN systems to adapt to the oxidative stress, the cytosolic TXN reductase 1 (TXNRD1) is a vital part of thioredoxin (TXN) system, which is up-regulated in various cancers(Cadenas et al. 2010; Hughes et al. 2018; Lincoln et al. 2003) and high expression is associated with poor clinical prognosis in multiply types of cancers.(Bhatia et al. 2016; Leone et al. 2017) In addition, TXNRD1 is also consider as a drug target with high efficacy and low toxicity. Recently, several studies found that the expression of TXNRD1 is high(Cadenas et al. 2010; Cho et al. 2019; Fu et al. 2017) and is related with poorer clinicopathological features, meantime, inhibition of TXNRD1 Inhibits the development and progression of hepatocellular carcinoma cells.(Lee et al. 2019a) UCK2 gene encodes uridine-cytidine kinase 2, which plays vital role in biosynthesis of the pyrimidine nucleotide.(Schumacher et al. 2013; Tomoike et al. 2017) It have reported that UCK2 is overexpressed in multiple type of cancers and is associated with poor prognosis, including breast cancer, pancreatic cancer, colon cancer. Yu S et al(Yu et al. 2019) showed that high UCK2 expression is associated with clinicopathologic feature and is a independent marker for predicting OS and RFS in hepatocellular carcinoma, the expression level may be influenced by the methylation of status cg0927774. In addition, knockdown of UCK2 suppressed proliferation, migration and invasion,(Huang et al. 2019) UCK2 may promotes HCC cell progress through stat3 signaling pathway. The alterations of membrane phospholipid levels could influence membrane fluidity and facilitate metastases because they affect motility, basement membrane invasion, and adhesion.(Taraboletti et al. 1989) LPCAT1 is a cytosolic enzyme that converts lysophosphatidylcholine (LPC) to phosphatidylcholine (PC). As an important subtype belongs to the 4 LPCAT subtypes,(Shindou \& Shimizu 2009) LPCAT1 has been obtained much attention for cancers, $L P C A T 1$ could contribute the progression, metastasis, and recurrence of cancer. To date, LPCAT1 over-expression have been reported in multiple types of cancers, including cell renal cell carcinoma,(Du et al. 2017) gastric cancer,(Uehara et al. 2016) breast cancer,(Abdelzaher \& Mostafa 2015) oral squamous cell carcinoma,(Shida-Sakazume et al. 2015) hepatocellular carcinoma.(Morita et al. 2013) The DNA primase polypeptide 1 (PRIMI) is responsible for synthesizing small RNA primers for Okazaki fragments generated during discontinuous DNA replication, the DNA replication cannot proceed without the catalytic function of PRIM1, thus 
452

453

454

455

456

457

458

459

460

461

462

463

464

465

466

467

468

469

470

471

472

473

474

475

476

477

478

479

480

481

482

483

484

485

486

487

488

489

490

491

492

PRIM1 is an vital role in the initiation (priming) of the DNA replication, and its aberrations may play a key tumorigenic factor by affecting the cell cycle transition from G1 to S phase.(Yotov et al. 1999) So far, PRIMI have been reported be associated with the formation of breast cancer.(Lee et al. 2019b)

Several significant biological process and signaling pathway which were active in high risk or low risk group have been revealed to provide a new insights of the development of HCC. We have divided patients from training cohort into two subtypes by performing our gene signature. Next, we conducted differential expression analysis between high and low group to the association between the metabolic genes and two subtypes. We found that upregulated genes in high risk group were mainly involved in cell cycle regulation, and upregulated genes in low risk group were mainly involved metabolic and energy regulation. Likely, GSEA analysis have demonstrated the similar result. Significant pathways which were active in high risk group were mainly related with cell cycle regulation. In contrast, meaningful pathways which were active in low risk group were mainly associated with metabolic and energy regulation.

\section{Conclusion}

The study identified ten prognostic genes that participate aberrant metabolism in HCC, and developed a metabolic ten-gene signature which provides more powerful prognostic information and improve the survival prediction for HCC. We could also score each patient according to the metabolic nine-gene signature and thus identified high-risk HCC patients. The prognostic ability of ten-gene signature was validated by internal testing cohort, GSE14520 testing cohort, ICGC testing cohort, entire testing cohort. Moreover, several significant biological process and signaling pathway have been identified to provide a new insights of the development of HCC. However, further biological experiments should performed to validate our results.

\section{Acknowledgements}

We are grateful to the reviewers for their constructive comments which led to improvements in this manuscript. In addition, thanks to Bin Zhao (Official Wechat Account: Bio_Med2017) of Xiamen University and Hexin Lin of Fujian Medical University for suggestions on the manuscripts.

\section{Reference}

Abdelzaher E, and Mostafa MF. 2015. Lysophosphatidylcholine acyltransferase 1 (LPCAT1) upregulation in breast carcinoma contributes to tumor progression and predicts early tumor recurrence. Tumour Biol 36:5473-5483. 10.1007/s13277-015-3214-8

Aoki T, and Weber G. 1981. Carbamoyl phosphate synthetase (glutamine-hydrolyzing): increased activity in cancer cells. Science 212:463-465. 10.1126/science.7209543

Arner ES, and Holmgren A. 2006. The thioredoxin system in cancer. Semin Cancer Biol 16:420-426. 10.1016/j.semcancer.2006.10.009

Aye Y, Li M, Long MJ, and Weiss RS. 2015. Ribonucleotide reductase and cancer: biological mechanisms and targeted therapies. Oncogene 34:2011-2021. 10.1038/onc.2014.155

Barajas JM, Reyes R, Guerrero MJ, Jacob ST, Motiwala T, and Ghoshal K. 2018. The role of miR-122 in the dysregulation of glucose-6-phosphate dehydrogenase (G6PD) expression in hepatocellular cancer. Sci Rep 8:9105. 10.1038/s41598-018-27358-5 
Benfeitas R, Bidkhori G, Mukhopadhyay B, Klevstig M, Arif M, Zhang C, Lee S, Cinar R, Nielsen J, Uhlen M, Boren J, Kunos G, and Mardinoglu A. 2019. Characterization of heterogeneous redox responses in hepatocellular carcinoma patients using network analysis. EBioMedicine 40:471-487. 10.1016/j.ebiom.2018.12.057

Bhatia M, McGrath KL, Di Trapani G, Charoentong P, Shah F, King MM, Clarke FM, and Tonissen KF. 2016. The thioredoxin system in breast cancer cell invasion and migration. Redox Biol 8:68-78. 10.1016/j.redox.2015.12.004

Bidkhori G, Benfeitas R, Klevstig M, Zhang C, Nielsen J, Uhlen M, Boren J, and Mardinoglu A. 2018. Metabolic network-based stratification of hepatocellular carcinoma reveals three distinct tumor subtypes. Proc Natl Acad Sci U S A 115:E11874-E11883. 10.1073/pnas.1807305115

Bray F, Ferlay J, Soerjomataram I, Siegel RL, Torre LA, and Jemal A. 2018. Global cancer statistics 2018: GLOBOCAN estimates of incidence and mortality worldwide for 36 cancers in 185 countries. CA Cancer $J$ Clin 68:394-424. 10.3322/caac.21492

Cadenas C, Franckenstein D, Schmidt M, Gehrmann M, Hermes M, Geppert B, Schormann W, Maccoux LJ, Schug M, Schumann A, Wilhelm C, Freis E, Ickstadt K, Rahnenfuhrer J, Baumbach JI, Sickmann A, and Hengstler JG. 2010. Role of thioredoxin reductase 1 and thioredoxin interacting protein in prognosis of breast cancer. Breast Cancer Res 12:R44. 10.1186/bcr2599

Caspi R, Billington R, Ferrer L, Foerster H, Fulcher CA, Keseler IM, Kothari A, Krummenacker M, Latendresse M, Mueller LA, Ong Q, Paley S, Subhraveti P, Weaver DS, and Karp PD. 2016. The MetaCyc database of metabolic pathways and enzymes and the BioCyc collection of pathway/genome databases. Nucleic Acids Res 44:D471-480. 10.1093/nar/gkv1164

Chakrabarti G. 2015. Mutant KRAS associated malic enzyme 1 expression is a predictive marker for radiation therapy response in non-small cell lung cancer. Radiat Oncol 10:145. 10.1186/s13014-015-0457-x

Chen CW, Li Y, Hu S, Zhou W, Meng Y, Li Z, Zhang Y, Sun J, Bo Z, DePamphilis ML, Yen Y, Han Z, and Zhu W. 2019. DHS (trans-4,4'-dihydroxystilbene) suppresses DNA replication and tumor growth by inhibiting RRM2 (ribonucleotide reductase regulatory subunit M2). Oncogene 38:2364-2379. 10.1038/s41388-0180584-6

Chen MC, Zhou B, Zhang K, Yuan YC, Un F, Hu S, Chou CM, Chen CH, Wu J, Wang Y, Liu X, Smith DL, Li H, Liu Z, Warden CD, Su L, Malkas LH, Chung YM, Hu MC, and Yen Y. 2015. The Novel Ribonucleotide Reductase Inhibitor COH29 Inhibits DNA Repair In Vitro. Mol Pharmacol 87:996-1005. 10.1124/mol.114.094987

Chiu DK, Tse AP, Xu IM, Di Cui J, Lai RK, Li LL, Koh HY, Tsang FH, Wei LL, Wong CM, Ng IO, and Wong CC. 2017. Hypoxia inducible factor HIF-1 promotes myeloid-derived suppressor cells accumulation through ENTPD2/CD39L1 in hepatocellular carcinoma. Nat Commun 8:517. 10.1038/s41467-017-00530-7

Cho SY, Kim S, Son MJ, Rou WS, Kim SH, Eun HS, and Lee BS. 2019. Clinical Significance of the Thioredoxin System and Thioredoxin-Domain-Containing Protein Family in Hepatocellular Carcinoma. Dig Dis Sci 64:123-136. 10.1007/s10620-018-5307-x

Das B, Roy J, Jain N, and Mallick B. 2019. Tumor suppressive activity of PIWI-interacting RNA in human fibrosarcoma mediated through repression of RRM2. Mol Carcinog 58:344-357. 10.1002/mc.22932

Dawany NB, Dampier WN, and Tozeren A. 2011. Large-scale integration of microarray data reveals genes and pathways common to multiple cancer types. Int J Cancer 128:2881-2891. 10.1002/ijc.25854

Du Y, Wang Q, Zhang X, Wang X, Qin C, Sheng Z, Yin H, Jiang C, Li J, and Xu T. 2017. Lysophosphatidylcholine 
acyltransferase 1 upregulation and concomitant phospholipid alterations in clear cell renal cell carcinoma. $J$ Exp Clin Cancer Res 36:66. 10.1186/s13046-017-0525-1

Fairbanks LD, Bofill M, Ruckemann K, and Simmonds HA. 1995. Importance of ribonucleotide availability to proliferating T-lymphocytes from healthy humans. Disproportionate expansion of pyrimidine pools and contrasting effects of de novo synthesis inhibitors. J Biol Chem 270:29682-29689.

Fu B, Meng W, Zeng X, Zhao H, Liu W, and Zhang T. 2017. TXNRD1 Is an Unfavorable Prognostic Factor for Patients with Hepatocellular Carcinoma. Biomed Res Int 2017:4698167. 10.1155/2017/4698167

Gao J, Chen H, Yu Y, Song J, Song H, Su X, Li W, Tong X, Qian W, Wang H, Dai J, and Guo Y. 2013. Inhibition of hepatocellular carcinoma growth using immunoliposomes for co-delivery of adriamycin and ribonucleotide reductase M2 siRNA. Biomaterials 34:10084-10098. 10.1016/j.biomaterials.2013.08.088

Gao Y, Wang X, Sang Z, Li Z, Liu F, Mao J, Yan D, Zhao Y, Wang H, Li P, Ying X, Zhang X, He K, and Wang H. 2017. Quantitative proteomics by SWATH-MS reveals sophisticated metabolic reprogramming in hepatocellular carcinoma tissues. Sci Rep 7:45913. 10.1038/srep45913

Goan YG, Zhou B, Hu E, Mi S, and Yen Y. 1999. Overexpression of ribonucleotide reductase as a mechanism of resistance to 2,2-difluorodeoxycytidine in the human KB cancer cell line. Cancer Res 59:4204-4207.

Hanahan D, and Weinberg RA. 2011. Hallmarks of cancer: the next generation. Cell 144:646-674. 10.1016/j.cell.2011.02.013

Hanazaki K, Kajikawa S, Koide N, Adachi W, and Amano J. 2001. Prognostic factors after hepatic resection for hepatocellular carcinoma with hepatitis $\mathrm{C}$ viral infection: univariate and multivariate analysis. $\mathrm{Am} J$ Gastroenterol 96:1243-1250. 10.1111/j.1572-0241.2001.03634.x

He B, Yin J, Gong S, Gu J, Xiao J, Shi W, Ding W, and He Y. 2017. Bioinformatics analysis of key genes and pathways for hepatocellular carcinoma transformed from cirrhosis. Medicine (Baltimore) 96:e6938. 10.1097/MD.0000000000006938

Hsieh YY, Chou CJ, Lo HL, and Yang PM. 2016. Repositioning of a cyclin-dependent kinase inhibitor GW8510 as a ribonucleotide reductase M2 inhibitor to treat human colorectal cancer. Cell Death Discov 2:16027. 10.1038/cddiscovery.2016.27

Huang S, Li J, Tam NL, Sun C, Hou Y, Hughes B, Wang Z, Zhou Q, He X, and Wu L. 2019. Uridine-cytidine kinase 2 upregulation predicts poor prognosis of hepatocellular carcinoma and is associated with cancer aggressiveness. Mol Carcinog 58:603-615. 10.1002/mc.22954

Hughes DJ, Kunicka T, Schomburg L, Liska V, Swan N, and Soucek P. 2018. Expression of Selenoprotein Genes and Association with Selenium Status in Colorectal Adenoma and Colorectal Cancer. Nutrients 10. 10.3390/nu10111812

Jiang L, Zhao L, Bi J, Guan Q, Qi A, Wei Q, He M, Wei M, and Zhao L. 2019. Glycolysis gene expression profilings screen for prognostic risk signature of hepatocellular carcinoma. Aging (Albany NY) 11:10861-10882. 10.18632/aging. 102489

Khan S, Abdelrahim M, Samudio I, and Safe S. 2003. Estrogen receptor/Sp1 complexes are required for induction of cad gene expression by 17beta-estradiol in breast cancer cells. Endocrinology 144:2325-2335. 10.1210/en.2002-0149

Kim H, Kelly RE, and Evans DR. 1992. The structural organization of the hamster multifunctional protein CAD. Controlled proteolysis, domains, and linkers. J Biol Chem 267:7177-7184.

Knoblich K, Wang HX, Sharma C, Fletcher AL, Turley SJ, and Hemler ME. 2014. Tetraspanin TSPAN12 regulates 
tumor growth and metastasis and inhibits beta-catenin degradation. Cell Mol Life Sci 71:1305-1314. 10.1007/s00018-013-1444-8

Kolberg M, Bruun J, Murumagi A, Mpindi JP, Bergsland CH, Holand M, Eilertsen IA, Danielsen SA, Kallioniemi O, and Lothe RA. 2017. Drug sensitivity and resistance testing identifies PLK1 inhibitors and gemcitabine as potent drugs for malignant peripheral nerve sheath tumors. Mol Oncol 11:1156-1171. 10.1002/18780261.12086

Kosakowska-Cholody T, Cholody WM, Hariprakasha HK, Monks A, Kar S, Wang M, Michejda CJ, and Carr BI. 2009. Growth inhibition of hepatocellular carcinoma cells in vitro and in vivo by the 8-methoxy analog of WMC79. Cancer Chemother Pharmacol 63:769-778. 10.1007/s00280-008-0801-z

Lee B, Ha SY, Song DH, Lee HW, Cho SY, and Park CK. 2014. High expression of ribonucleotide reductase subunit M2 correlates with poor prognosis of hepatocellular carcinoma. Gut Liver 8:662-668. 10.5009/gnl13392

Lee D, Xu IM, Chiu DK, Leibold J, Tse AP, Bao MH, Yuen VW, Chan CY, Lai RK, Chin DW, Chan DF, Cheung TT, Chok SH, Wong CM, Lowe SW, Ng IO, and Wong CC. 2019a. Induction of Oxidative Stress Through Inhibition of Thioredoxin Reductase 1 Is an Effective Therapeutic Approach for Hepatocellular Carcinoma. Hepatology 69:1768-1786. 10.1002/hep.30467

Lee WH, Chen LC, Lee CJ, Huang CC, Ho YS, Yang PS, Ho CT, Chang HL, Lin IH, Chang HW, Liu YR, Wu CH, and Tu SH. 2019b. DNA primase polypeptide 1 (PRIM1) involves in estrogen-induced breast cancer formation through activation of the G2/M cell cycle checkpoint. Int $J$ Cancer 144:615-630. 10.1002/ijc. 31788

Leone A, Roca MS, Ciardiello C, Costantini S, and Budillon A. 2017. Oxidative Stress Gene Expression Profile Correlates with Cancer Patient Poor Prognosis: Identification of Crucial Pathways Might Select Novel Therapeutic Approaches. Oxid Med Cell Longev 2017:2597581. 10.1155/2017/2597581

Li B, Feng W, Luo O, Xu T, Cao Y, Wu H, Yu D, and Ding Y. 2017. Development and Validation of a Three-gene Prognostic Signature for Patients with Hepatocellular Carcinoma. Sci Rep 7:5517. 10.1038/s41598-017$04811-5$

Li C, Ruan HQ, Liu YS, Xu MJ, Dai J, Sheng QH, Tan YX, Yao ZZ, Wang HY, Wu JR, and Zeng R. 2012. Quantitative proteomics reveal up-regulated protein expression of the SET complex associated with hepatocellular carcinoma. J Proteome Res 11:871-885. 10.1021/pr2006999

Liang WH, Li N, Yuan ZQ, Qian XL, and Wang ZH. 2019. DSCAM-AS1 promotes tumor growth of breast cancer by reducing miR-204-5p and up-regulating RRM2. Mol Carcinog 58:461-473. 10.1002/mc.22941

Liao R, Ren G, Liu H, Chen X, Cao Q, Wu X, Li J, and Dong C. 2018. ME1 promotes basal-like breast cancer progression and associates with poor prognosis. Sci Rep 8:16743. 10.1038/s41598-018-35106-y

Lincoln DT, Ali Emadi EM, Tonissen KF, and Clarke FM. 2003. The thioredoxin-thioredoxin reductase system: overexpression in human cancer. Anticancer Res 23:2425-2433.

Liu M, Chen Y, Huang B, Mao S, Cai K, Wang L, and Yao X. 2018. Tumor-suppressing effects of microRNA-612 in bladder cancer cells by targeting malic enzyme 1 expression. Int $J$ Oncol 52:1923-1933. 10.3892/ijo.2018.4342

Liu X, Zhang H, Lai L, Wang X, Loera S, Xue L, He H, Zhang K, Hu S, Huang Y, Nelson RA, Zhou B, Zhou L, Chu P, Zhang S, Zheng S, and Yen Y. 2013a. Ribonucleotide reductase small subunit M2 serves as a prognostic biomarker and predicts poor survival of colorectal cancers. Clin Sci (Lond) 124:567-578. $10.1042 / \mathrm{CS} 20120240$ 
616

617

618

619

620

621

622

623

624

625

626

627

628

629

630

631

632

633

634

635

636

637

638

639

640

641

642

643

644

645

646

647

648

649

650

651

652

653

654

655

656

Liu Y, Marks K, Cowley GS, Carretero J, Liu Q, Nieland TJ, Xu C, Cohoon TJ, Gao P, Zhang Y, Chen Z, Altabef AB, Tchaicha JH, Wang X, Choe S, Driggers EM, Zhang J, Bailey ST, Sharpless NE, Hayes DN, Patel NM, Janne PA, Bardeesy N, Engelman JA, Manning BD, Shaw RJ, Asara JM, Scully R, Kimmelman A, Byers LA, Gibbons DL, Wistuba, II, Heymach JV, Kwiatkowski DJ, Kim WY, Kung AL, Gray NS, Root DE, Cantley LC, and Wong KK. 2013b. Metabolic and functional genomic studies identify deoxythymidylate kinase as a target in LKB1-mutant lung cancer. Cancer Discov 3:870-879. 10.1158/2159-8290.CD-13-0015

Long J, Zhang L, Wan X, Lin J, Bai Y, Xu W, Xiong J, and Zhao H. 2018. A four-gene-based prognostic model predicts overall survival in patients with hepatocellular carcinoma. J Cell Mol Med 22:5928-5938. 10.1111/jcmm.13863

Lu AG, Feng H, Wang PX, Han DP, Chen XH, and Zheng MH. 2012. Emerging roles of the ribonucleotide reductase M2 in colorectal cancer and ultraviolet-induced DNA damage repair. World J Gastroenterol 18:4704-4713. 10.3748/wjg.v18.i34.4704

Lu YX, Ju HQ, Liu ZX, Chen DL, Wang Y, Zhao Q, Wu QN, Zeng ZL, Qiu HB, Hu PS, Wang ZQ, Zhang DS, Wang F, and $\mathrm{Xu}$ RH. 2018. ME1 Regulates NADPH Homeostasis to Promote Gastric Cancer Growth and Metastasis. Cancer Res 78:1972-1985. 10.1158/0008-5472.CAN-17-3155

Mann CD, Neal CP, Garcea G, Manson MM, Dennison AR, and Berry DP. 2007. Prognostic molecular markers in hepatocellular carcinoma: a systematic review. Eur J Cancer 43:979-992. 10.1016/j.ejca.2007.01.004

Minami K, Shinsato Y, Yamamoto M, Takahashi H, Zhang S, Nishizawa Y, Tabata S, Ikeda R, Kawahara K, Tsujikawa K, Chijiiwa K, Yamada K, Akiyama S, Perez-Torras S, Pastor-Anglada M, Furukawa T, and Yasuo T. 2015. Ribonucleotide reductase is an effective target to overcome gemcitabine resistance in gemcitabine-resistant pancreatic cancer cells with dual resistant factors. J Pharmacol Sci 127:319-325. 10.1016/j.jphs.2015.01.006

Morikawa T, Hino R, Uozaki H, Maeda D, Ushiku T, Shinozaki A, Sakatani T, and Fukayama M. 2010a. Expression of ribonucleotide reductase M2 subunit in gastric cancer and effects of RRM2 inhibition in vitro. Hum Pathol 41:1742-1748. 10.1016/j.humpath.2010.06.001

Morikawa T, Maeda D, Kume H, Homma Y, and Fukayama M. 2010b. Ribonucleotide reductase M2 subunit is a novel diagnostic marker and a potential therapeutic target in bladder cancer. Histopathology 57:885-892. 10.1111/j.1365-2559.2010.03725.x

Morin A, Fritsch L, Mathieu JR, Gilbert C, Guarmit B, Firlej V, Gallou-Kabani C, Vieillefond A, Delongchamps NB, and Cabon F. 2012. Identification of CAD as an androgen receptor interactant and an early marker of prostate tumor recurrence. FASEB J 26:460-467. 10.1096/fj.11-191296

Morita Y, Sakaguchi T, Ikegami K, Goto-Inoue N, Hayasaka T, Hang VT, Tanaka H, Harada T, Shibasaki Y, Suzuki A, Fukumoto K, Inaba K, Murakami M, Setou M, and Konno H. 2013. Lysophosphatidylcholine acyltransferase 1 altered phospholipid composition and regulated hepatoma progression. J Hepatol 59:292299. 10.1016/j.jhep.2013.02.030

Munemoto M, Mukaisho KI, Miyashita T, Oyama K, Haba Y, Okamoto K, Kinoshita J, Ninomiya I, Fushida S, Taniura N, Sugihara H, and Fujimura T. 2019. Roles of the hexosamine biosynthetic pathway and pentose phosphate pathway in bile acid-induced cancer development. Cancer Sci 110:2408-2420. 10.1111/cas.14105

Nakano Y, Tanno S, Koizumi K, Nishikawa T, Nakamura K, Minoguchi M, Izawa T, Mizukami Y, Okumura T, and Kohgo Y. 2007. Gemcitabine chemoresistance and molecular markers associated with gemcitabine transport and metabolism in human pancreatic cancer cells. Br J Cancer 96:457-463. 10.1038/sj.bjc.6603559

Peer) reviewing PDF | (2020:01:45125:1:1:NEW 6 Apr 2020) 
657

658

659

660

661

662

663

664

665

666

667

668

669

670

671

672

673

674

675

676

677

678

679

680

681

682

683

684

685

686

687

688

689

690

691

692

693

694

695

696

697

Ohl F, Jung M, Radonic A, Sachs M, Loening SA, and Jung K. 2006. Identification and validation of suitable endogenous reference genes for gene expression studies of human bladder cancer. $J$ Urol 175:1915-1920. 10.1016/S0022-5347(05)00919-5

Pu H, Zhang Q, Zhao C, Shi L, Wang Y, Wang J, and Zhang M. 2015. Overexpression of G6PD is associated with high risks of recurrent metastasis and poor progression-free survival in primary breast carcinoma. World $J$ Surg Oncol 13:323. 10.1186/s12957-015-0733-0

Qiao GJ, Chen L, Wu JC, and Li ZR. 2019. Identification of an eight-gene signature for survival prediction for patients with hepatocellular carcinoma based on integrated bioinformatics analysis. PeerJ 7:e6548. 10.7717/peerj.6548

Sacoman JL, Badish LN, Sharkey TD, and Hollingsworth RI. 2012. The metabolic and biochemical impact of glucose 6-sulfonate (sulfoquinovose), a dietary sugar, on carbohydrate metabolism. Carbohydr Res 362:21-29. 10.1016/j.carres.2012.09.014

Schumacher FR, Wang Z, Skotheim RI, Koster R, Chung CC, Hildebrandt MA, Kratz CP, Bakken AC, Bishop DT, Cook MB, Erickson RL, Fossa SD, Greene MH, Jacobs KB, Kanetsky PA, Kolonel LN, Loud JT, Korde LA, Le Marchand L, Lewinger JP, Lothe RA, Pike MC, Rahman N, Rubertone MV, Schwartz SM, Siegmund KD, Skinner EC, Turnbull C, Van Den Berg DJ, Wu X, Yeager M, Nathanson KL, Chanock SJ, Cortessis VK, and McGlynn KA. 2013. Testicular germ cell tumor susceptibility associated with the UCK2 locus on chromosome 1q23. Hum Mol Genet 22:2748-2753. 10.1093/hmg/ddt109

Shah KN, Mehta KR, Peterson D, Evangelista M, Livesey JC, and Faridi JS. 2014. AKT-induced tamoxifen resistance is overturned by RRM2 inhibition. Mol Cancer Res 12:394-407. 10.1158/1541-7786.MCR-13-0219

Shida-Sakazume T, Endo-Sakamoto Y, Unozawa M, Fukumoto C, Shimada K, Kasamatsu A, Ogawara K, Yokoe H, Shiiba M, Tanzawa H, and Uzawa K. 2015. Lysophosphatidylcholine acyltransferase1 overexpression promotes oral squamous cell carcinoma progression via enhanced biosynthesis of platelet-activating factor. PLoS One 10:e0120143. 10.1371/journal.pone.0120143

Shindou H, and Shimizu T. 2009. Acyl-CoA:lysophospholipid acyltransferases. J Biol Chem 284:1-5. 10.1074/jbc.R800046200

Sigoillot FD, Sigoillot SM, and Guy HI. 2004. Breakdown of the regulatory control of pyrimidine biosynthesis in human breast cancer cells. Int J Cancer 109:491-498. 10.1002/ijc.11717

Singhal A, Jayaraman M, Dhanasekaran DN, and Kohli V. 2012. Molecular and serum markers in hepatocellular carcinoma: predictive tools for prognosis and recurrence. Crit Rev Oncol Hematol 82:116-140. 10.1016/j.critrevonc.2011.05.005

Souglakos J, Boukovinas I, Taron M, Mendez P, Mavroudis D, Tripaki M, Hatzidaki D, Koutsopoulos A, Stathopoulos E, Georgoulias V, and Rosell R. 2008. Ribonucleotide reductase subunits M1 and M2 mRNA expression levels and clinical outcome of lung adenocarcinoma patients treated with docetaxel/gemcitabine. Br J Cancer 98:1710-1715. 10.1038/sj.bjc.6604344

Sun Y, Gu X, Zhang E, Park MA, Pereira AM, Wang S, Morrison T, Li C, Blenis J, Gerbaudo VH, Henske EP, and Yu JJ. 2014. Estradiol promotes pentose phosphate pathway addiction and cell survival via reactivation of Akt in mTORC1 hyperactive cells. Cell Death Dis 5:e1231. 10.1038/cddis.2014.204

Suner A, Carr BI, Akkiz H, Karakulah G, Uskudar O, Yalcin K, Kuran S, Tokat Y, Yilmaz S, Ozakyol A, Tokmak S, Balli T, Yucesoy M, Bahceci HI, Ulku A, Akcam T, Polat KY, Ekinci N, Simsek H, Ormeci N, Sonsuz A, Demir M, Kilic M, Uygun A, Demir A, Delik A, Arslan B, Doran F, Altintas E, Temel T, and Bektas A.

Peer) reviewing PDF | (2020:01:45125:1:1:NEW 6 Apr 2020) 
2019a. C-Reactive Protein and Platelet-Lymphocyte Ratio as Potential Tumor Markers in Low-AlphaFetoprotein Hepatocellular Carcinoma. Oncology 96:25-32. 10.1159/000492473

Suner A, Carr BI, Akkiz H, Uskudar O, Kuran S, Tokat Y, Tokmak S, Balli T, Ulku A, AkCam T, Delik A, Arslan B, Doran F, YalCin K, Ekinci N, Yilmaz S, Ozakyol A, Yucesoy M, BahCeci HI, Polat KY, Simsek H, Ormeci N, Sonsuz A, Demir M, Kili CM, Uygun A, Demir A, Altintas E, Karakulah G, Temel T, and Bektas A. 2019b. Inflammatory markers C-reactive protein and PLR in relation to HCC characteristics. $J$ Transl Sci 5. $10.15761 / \mathrm{JTS} .1000260$

Tangkijvanich P, Anukulkarnkusol N, Suwangool P, Lertmaharit S, Hanvivatvong O, Kullavanijaya P, and Poovorawan Y. 2000. Clinical characteristics and prognosis of hepatocellular carcinoma: analysis based on serum alpha-fetoprotein levels. J Clin Gastroenterol 31:302-308. 10.1097/00004836-200012000-00007

Taraboletti G, Perin L, Bottazzi B, Mantovani A, Giavazzi R, and Salmona M. 1989. Membrane fluidity affects tumorcell motility, invasion and lung-colonizing potential. Int J Cancer 44:707-713. 10.1002/ijc.2910440426

Tomoike F, Nakagawa N, Fukui K, Yano T, Kuramitsu S, and Masui R. 2017. Indispensable residue for uridine binding in the uridine-cytidine kinase family. Biochem Biophys Rep 11:93-98. 10.1016/j.bbrep.2017.07.002

Uehara T, Kikuchi H, Miyazaki S, Ino I, Setoguchi T, Hiramatsu Y, Ohta M, Kamiya K, Morita Y, Tanaka H, Baba S, Hayasaka T, Setou M, and Konno H. 2016. Overexpression of Lysophosphatidylcholine Acyltransferase 1 and Concomitant Lipid Alterations in Gastric Cancer. Ann Surg Oncol 23 Suppl 2:S206-213. 10.1245/s10434-015-4459-6

Uhlen M, Fagerberg L, Hallstrom BM, Lindskog C, Oksvold P, Mardinoglu A, Sivertsson A, Kampf C, Sjostedt E, Asplund A, Olsson I, Edlund K, Lundberg E, Navani S, Szigyarto CA, Odeberg J, Djureinovic D, Takanen JO, Hober S, Alm T, Edqvist PH, Berling H, Tegel H, Mulder J, Rockberg J, Nilsson P, Schwenk JM, Hamsten M, von Feilitzen K, Forsberg M, Persson L, Johansson F, Zwahlen M, von Heijne G, Nielsen J, and Ponten F. 2015. Proteomics. Tissue-based map of the human proteome. Science 347:1260419. 10.1126/science. 1260419

Wang J, Yuan W, Chen Z, Wu S, Chen J, Ge J, Hou F, and Chen Z. 2012a. Overexpression of G6PD is associated with poor clinical outcome in gastric cancer. Tumour Biol 33:95-101. 10.1007/s13277-011-0251-9

Wang LM, Lu FF, Zhang SY, Yao RY, Xing XM, and Wei ZM. 2012b. Overexpression of catalytic subunit M2 in patients with ovarian cancer. Chin Med J (Engl) 125:2151-2156.

Wang X, Liu H, Zhang X, Li X, Gu H, Zhang H, and Fan R. 2016. G6PD downregulation triggered growth inhibition and induced apoptosis by regulating STAT3 signaling pathway in esophageal squamous cell carcinoma. Tumour Biol 37:781-789. 10.1007/s13277-015-3861-9

Wen D, Liu D, Tang J, Dong L, Liu Y, Tao Z, Wan J, Gao D, Wang L, Sun H, Fan J, and Wu W. 2015. Malic enzyme 1 induces epithelial-mesenchymal transition and indicates poor prognosis in hepatocellular carcinoma. Tumour Biol 36:6211-6221. 10.1007/s13277-015-3306-5

Woo SH, Yang LP, Chuang HC, Fitzgerald A, Lee HY, Pickering C, Myers JN, and Skinner HD. 2016. Downregulation of malic enzyme 1 and 2: Sensitizing head and neck squamous cell carcinoma cells to therapyinduced senescence. Head Neck 38 Suppl 1:E934-940. 10.1002/hed.24129

Wu M, Liu Z, Li X, Zhang A, Lin D, and Li N. 2019. Analysis of potential key genes in very early hepatocellular carcinoma. World J Surg Oncol 17:77. 10.1186/s12957-019-1616-6

Xiang XH, Yang L, Zhang X, Ma XH, Miao RC, Gu JX, Fu YN, Yao Q, Zhang JY, Liu C, Lin T, and Qu K. 2019. Seven-senescence-associated gene signature predicts overall survival for Asian patients with hepatocellular 
carcinoma. World J Gastroenterol 25:1715-1728. 10.3748/wjg.v25.i14.1715

Xu B, Wang F, Song C, Sun Z, Cheng K, Tan Y, Wang H, and Zou H. 2014. Large-scale proteome quantification of hepatocellular carcinoma tissues by a three-dimensional liquid chromatography strategy integrated with sample preparation. J Proteome Res 13:3645-3654. 10.1021/pr500200s

Xu XR, Huang J, Xu ZG, Qian BZ, Zhu ZD, Yan Q, Cai T, Zhang X, Xiao HS, Qu J, Liu F, Huang QH, Cheng ZH, Li NG, Du JJ, Hu W, Shen KT, Lu G, Fu G, Zhong M, Xu SH, Gu WY, Huang W, Zhao XT, Hu GX, Gu JR, Chen Z, and Han ZG. 2001. Insight into hepatocellular carcinogenesis at transcriptome level by comparing gene expression profiles of hepatocellular carcinoma with those of corresponding noncancerous liver. Proc Natl Acad Sci U S A 98:15089-15094. 10.1073/pnas.241522398

Yan Y, Lu Y, Mao K, Zhang M, Liu H, Zhou Q, Lin J, Zhang J, Wang J, and Xiao Z. 2019. Identification and validation of a prognostic four-genes signature for hepatocellular carcinoma: integrated ceRNA network analysis. Hepatol Int 13:618-630. 10.1007/s12072-019-09962-3

Yeh HW, Lee SS, Chang CY, Hu CM, and Jou YS. 2017. Pyrimidine metabolic rate limiting enzymes in poorlydifferentiated hepatocellular carcinoma are signature genes of cancer stemness and associated with poor prognosis. Oncotarget 8:77734-77751. 10.18632/oncotarget.20774

Yotov WV, Hamel H, Rivard GE, Champagne MA, Russo PA, Leclerc JM, Bernstein ML, and Levy E. 1999. Amplifications of DNA primase 1 (PRIM1) in human osteosarcoma. Genes Chromosomes Cancer 26:62-69. 10.1002/(sici)1098-2264(199909)26:1<62::aid-gcc9>3.0.co;2-f

Yu D, Zhuang L, Sun X, Chen J, Yao Y, Meng K, and Ding Y. 2007. Particular distribution and expression pattern of endoglin (CD105) in the liver of patients with hepatocellular carcinoma. BMC Cancer 7:122. 10.1186/14712407-7-122

Yu DC, Chen J, and Ding YT. 2010. Hypoxic and highly angiogenic non-tumor tissues surrounding hepatocellular carcinoma: the 'niche' of endothelial progenitor cells. Int J Mol Sci 11:2901-2909. 10.3390/ijms11082901

Yu R, Tan Z, Xiang X, Dan Y, and Deng G. 2017. Effectiveness of PIVKA-II in the detection of hepatocellular carcinoma based on real-world clinical data. BMC Cancer 17:608. 10.1186/s12885-017-3609-6

Yu S, Li X, Guo X, Zhang H, Qin R, and Wang M. 2019. UCK2 upregulation might serve as an indicator of unfavorable prognosis of hepatocellular carcinoma. IUBMB Life 71:105-112. 10.1002/iub.1941

Zhang X, Zhang X, Li Y, Shao Y, Xiao J, Zhu G, and Li F. 2017. PAK4 regulates G6PD activity by p53 degradation involving colon cancer cell growth. Cell Death Dis 8:e2820. 10.1038/cddis.2017.85

Zhao N, Cao J, Xu L, Tang Q, Dobrolecki LE, Lv X, Talukdar M, Lu Y, Wang X, Hu DZ, Shi Q, Xiang Y, Wang Y, Liu X, Bu W, Jiang Y, Li M, Gong Y, Sun Z, Ying H, Yuan B, Lin X, Feng XH, Hartig SM, Li F, Shen H, Chen Y, Han L, Zeng Q, Patterson JB, Kaipparettu BA, Putluri N, Sicheri F, Rosen JM, Lewis MT, and Chen X. 2018. Pharmacological targeting of MYC-regulated IRE1/XBP1 pathway suppresses MYC-driven breast cancer. J Clin Invest 128:1283-1299. 10.1172/JCI95873

Zheng FJ, Ye HB, Wu MS, Lian YF, Qian CN, and Zeng YX. 2012. Repressing malic enzyme 1 redirects glucose metabolism, unbalances the redox state, and attenuates migratory and invasive abilities in nasopharyngeal carcinoma cell lines. Chin J Cancer 31:519-531. 10.5732/cjc.012.10088

Zheng RS, Sun KX, Zhang SW, Zeng HM, Zou XN, Chen R, Gu XY, Wei WW, and He J. 2019. [Report of cancer epidemiology in China, 2015]. Zhonghua Zhong Liu Za Zhi 41:19-28. 10.3760/cma.j.issn.02533766.2019.01.005 


\section{Table $\mathbf{1}$ (on next page)}

Summary of patient demographics and clinical characteristics 
1 Table 1:Summary of patient demographics and clinical characteristics

\begin{tabular}{llllll}
\hline Characteristic & Training cohort & Internal testing cohort & GSE14520 testing cohort & ICGC testing cohort & Entire testing cohort \\
\hline Gender & & & & $182(75 \%)$ & $619(75 \%)$ \\
male & $246(67 \%)$ & $121(66 \%)$ & $191(87 \%)$ & $61(25 \%)$ & $209(25 \%)$ \\
female & $119(33 \%)$ & $63(34 \%)$ & $29(13 \%)$ & & $387(47 \%)$ \\
Age & & & $45(18 \%)$ & $441(53 \%)$ \\
$<60$ & $165(45 \%)$ & $83(45 \%)$ & $177(80 \%)$ & $198(82 \%)$ & $570(70 \%)$ \\
$>=60$ & $200(55 \%)$ & $101(55 \%)$ & $43(20 \%)$ & & $235(30 \%)$ \\
Stage & & & $146(60 \%)$ & $97(40 \%)$ \\
Stage I-II & $254(74 \%)$ & $136(78 \%)$ & $170(77 \%)$ & & $573(69 \%)$ \\
Stage III-IV & $88(26 \%)$ & $38(22 \%)$ & $50(23 \%)$ & $199(82 \%)$ & $255(31 \%)$ \\
Vital status & & & $45(18 \%)$ & \\
living & $239(65 \%)$ & $125(68 \%)$ & $135(61 \%)$ & \\
dead & $126(35 \%)$ & $59(32 \%)$ & $85(39 \%)$ & &
\end{tabular}


Table 2 (on next page)

Comparison of the AUC between gene signature and single gene 
1 Table 2: Comparison of the AUC between gene signature and single gene

\begin{tabular}{llllll}
\hline Characteristic & Training cohort & Internal testing cohort & GSE14520 testing cohort & ICGC testing cohort & Entire testing cohort \\
\hline Risk score & 0.786 & 0.773 & 0.707 & 0.775 & 0.732 \\
G6PD & 0.738 & 0.741 & 0.575 & 0.698 & 0.665 \\
LPCAT1 & 0.708 & 0.680 & 0.514 & 0.722 & 0.663 \\
ME1 & 0.630 & 0.642 & 0.514 & 0.597 & 0.586 \\
PRIM1 & 0.706 & 0.748 & 0.534 & 0.707 & 0.634 \\
RRM2 & 0.716 & 0.754 & 0.501 & 0.698 & 0.655 \\
TXNRD1 & 0.674 & 0.663 & 0.532 & 0.611 & 0.599 \\
UCK2 & 0.736 & 0.699 & 0.650 & 0.711 & 0.695 \\
CAD & 0.743 & 0.726 & 0.602 & 0.688 & 0.676 \\
DTYMK & 0.689 & 0.722 & 0.534 & 0.734 & 0.641 \\
ENTPD2 & 0.552 & 0.546 & 0.503 & 0.662 & 0.580 \\
\hline
\end{tabular}

2




\section{Figure 1}

\section{The schematic workflow of the study}

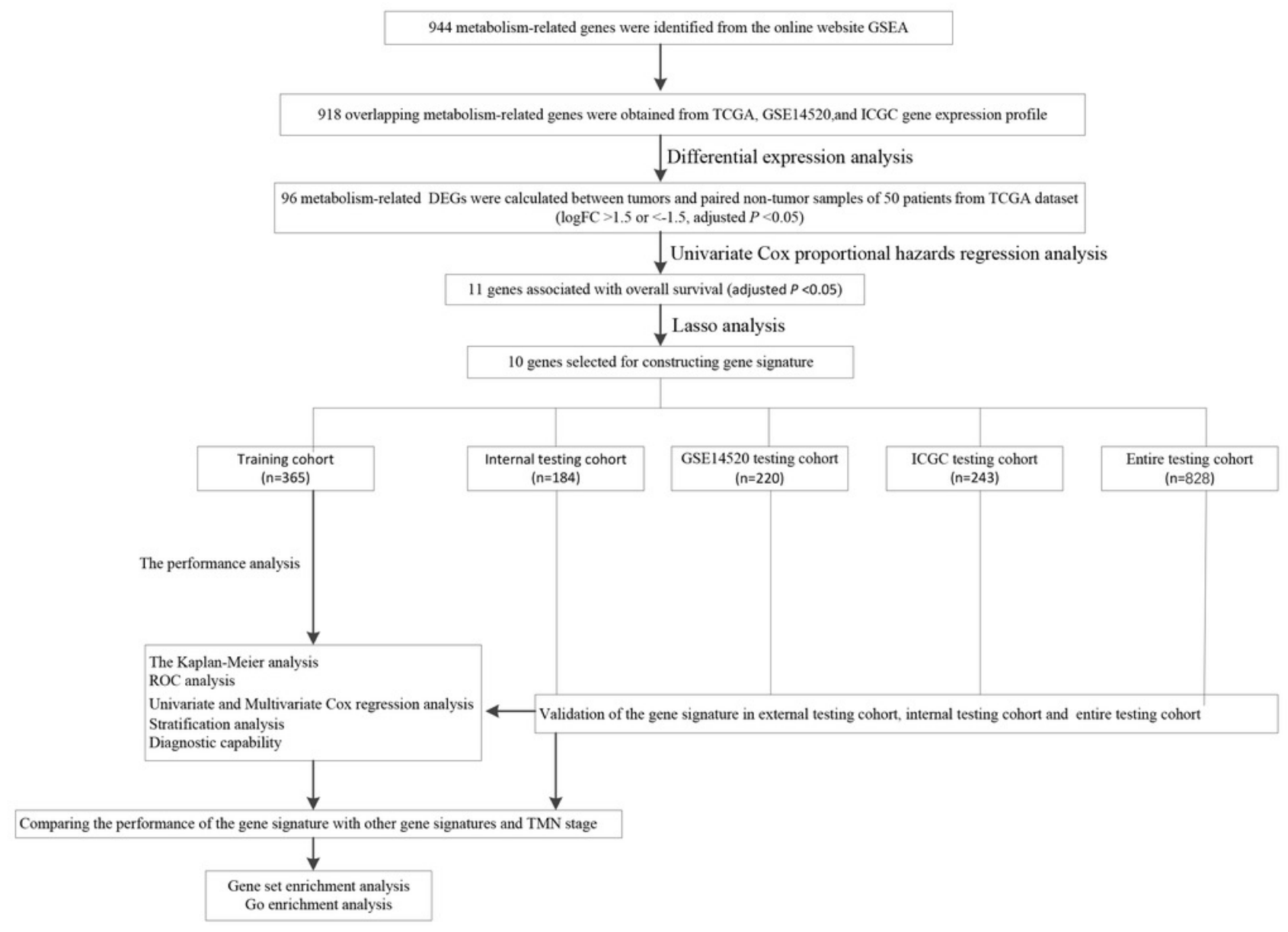




\section{Figure 2}

Heatmap and Volcano plot of metabolism-related DEGs

(A) Heatmap of metabolism-related DEGs. Red indicates that the gene expression is relatively high, green indicates that the gene expression is relatively low, and white indicates no significant changes in gene expression, (FDR $<0.05$, absolute $\log F C>1.5$ ). 10 prognostic genes were marked using red arrow. (B) Volcano plot of metabolism-related DEGs. The red points represent high expression genes, the green points represent low expression genes, the black points represent genes with no significant difference (FDR $<0.05$, absolute log FC>1.5). 10 prognostic genes were marked using black arrow.

A

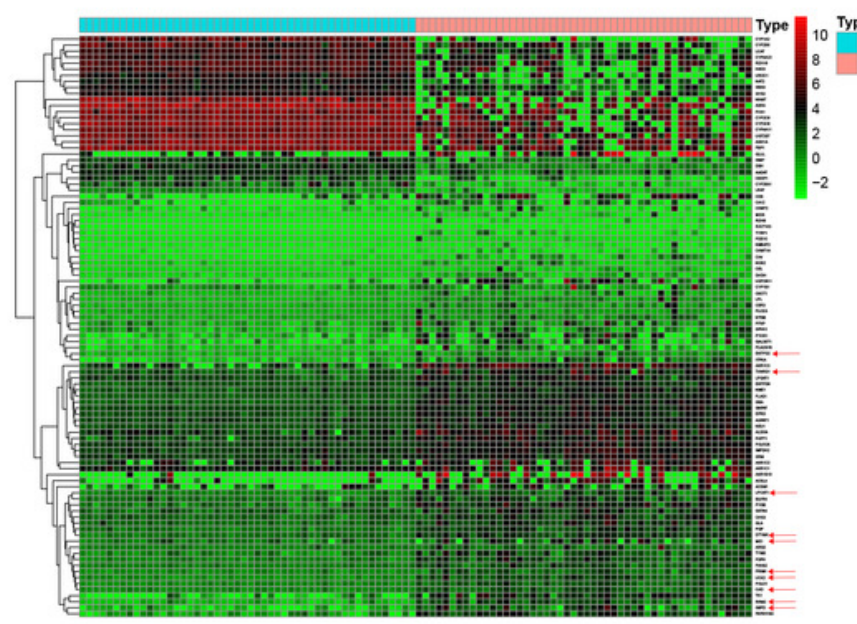

B

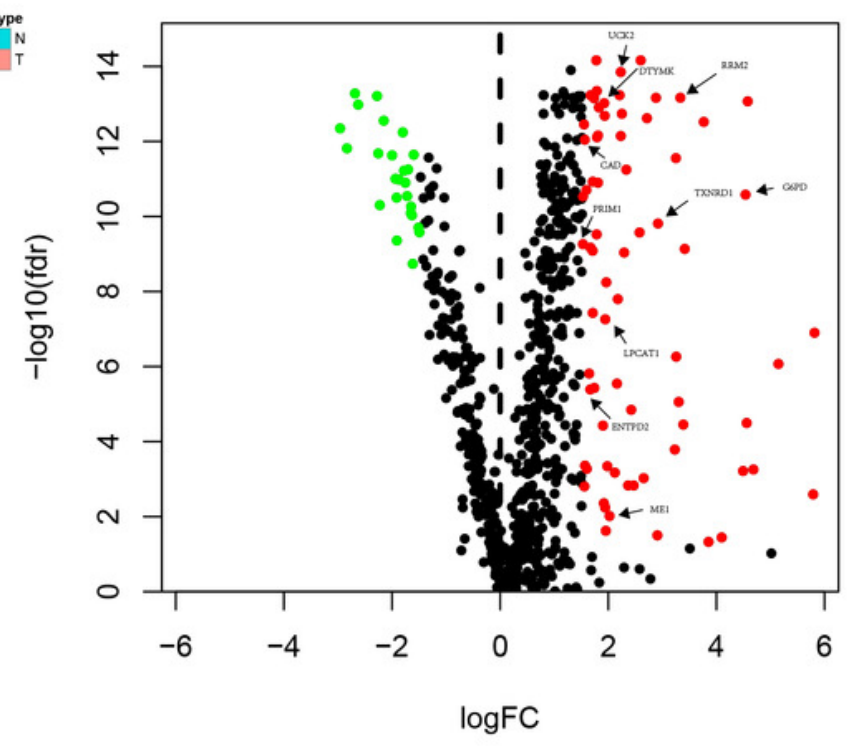


Figure 3

Expression of the ten genes in low- and high-risk groups of training cohort, internal testing cohort, GSE14520 testing cohort and ICGC testing cohort

(A)Training cohort (B) Internal testing cohort (C) GSE14520 testing cohort (D) ICGC testing cohort

A
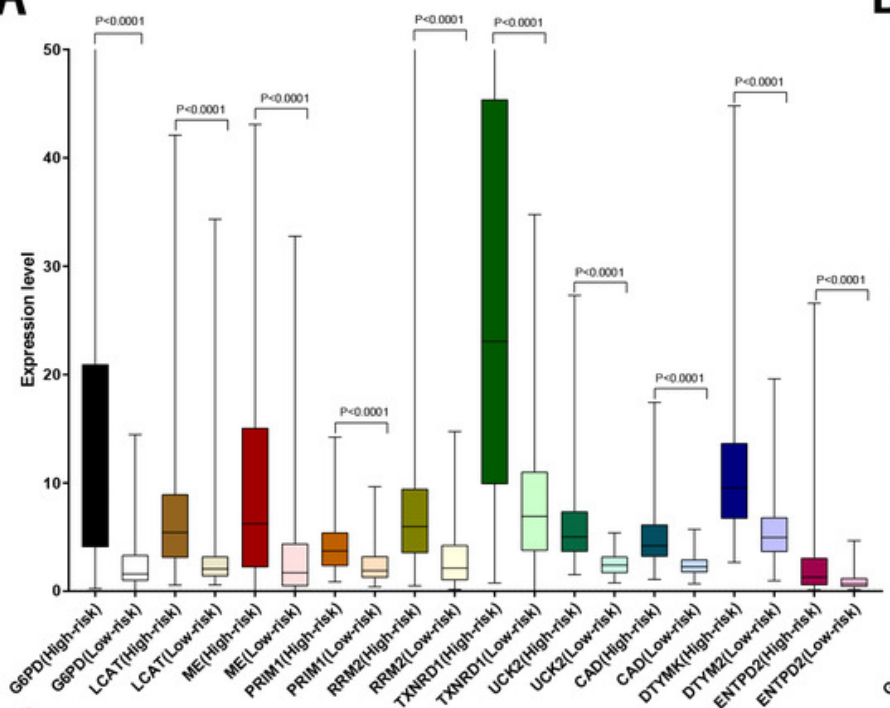

C

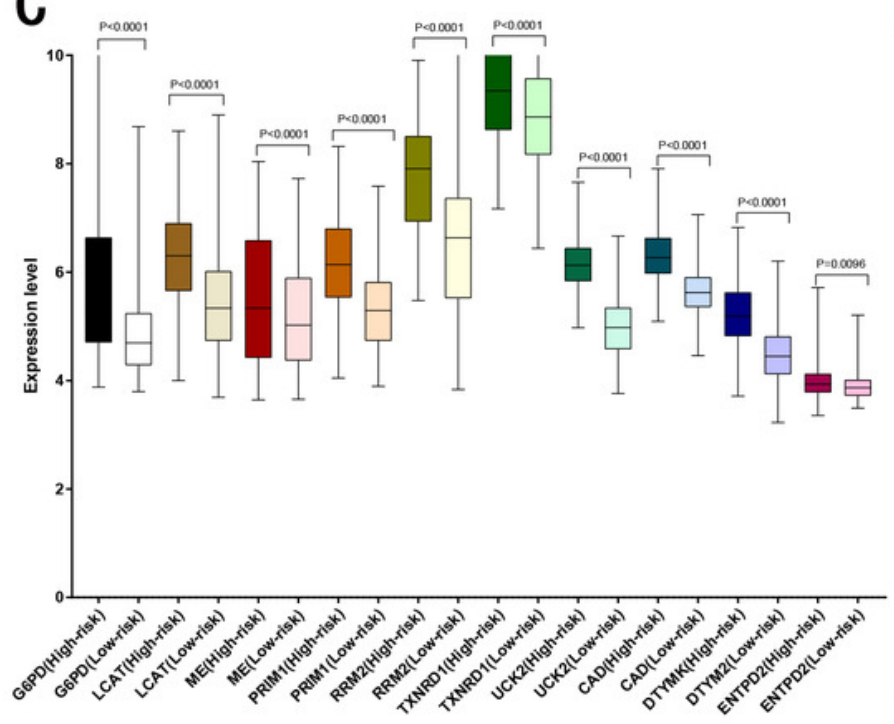

B

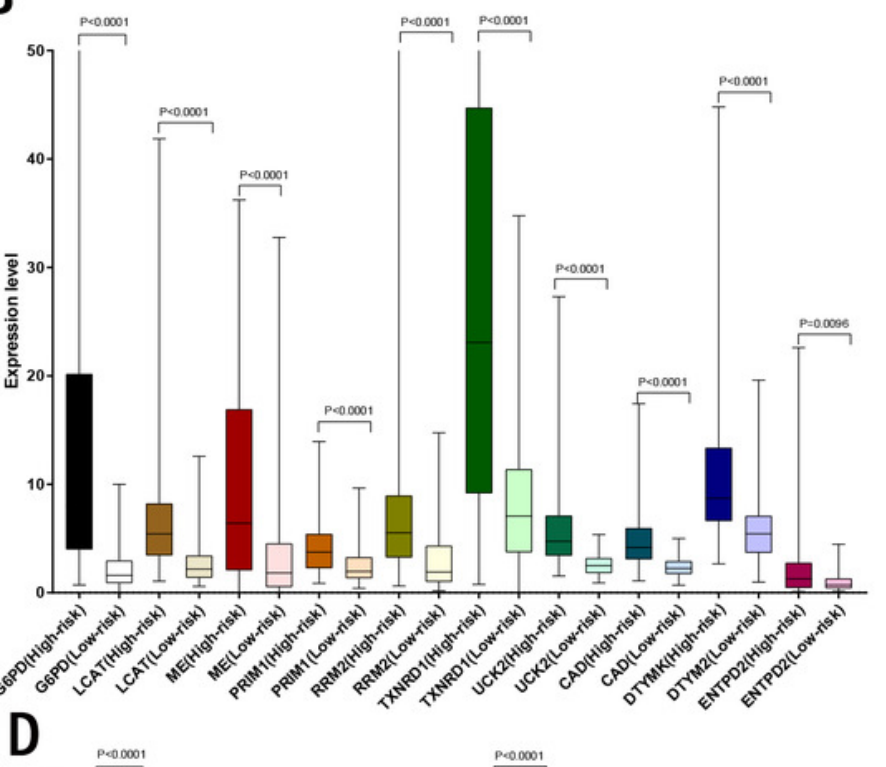

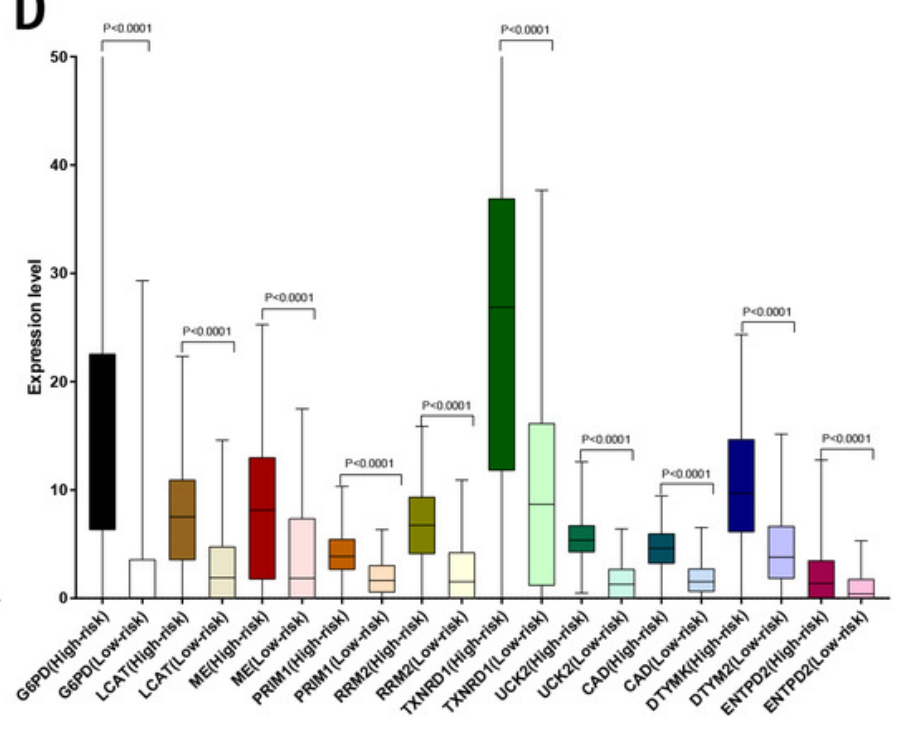




\section{Figure 4}

Gene signature performance analysis using training cohort

(A) Distribution of 10-gene-based risk scores, patient survival durations, gene expression levels (B) 1-year ROC curve analyses of gene signature and clinical parameters (C) 3-year ROC curve analyses of gene signature and clinical parameters (D) 5-year ROC curve analyses of gene signature and clinical parameters (E) Kaplan-Meier curves of OS based on gene signature (F) Prognostic value detection of the gene signature via univariate survival-related analysis (G) Prognostic value detection of the gene signature via multivariate survival-related analysis 

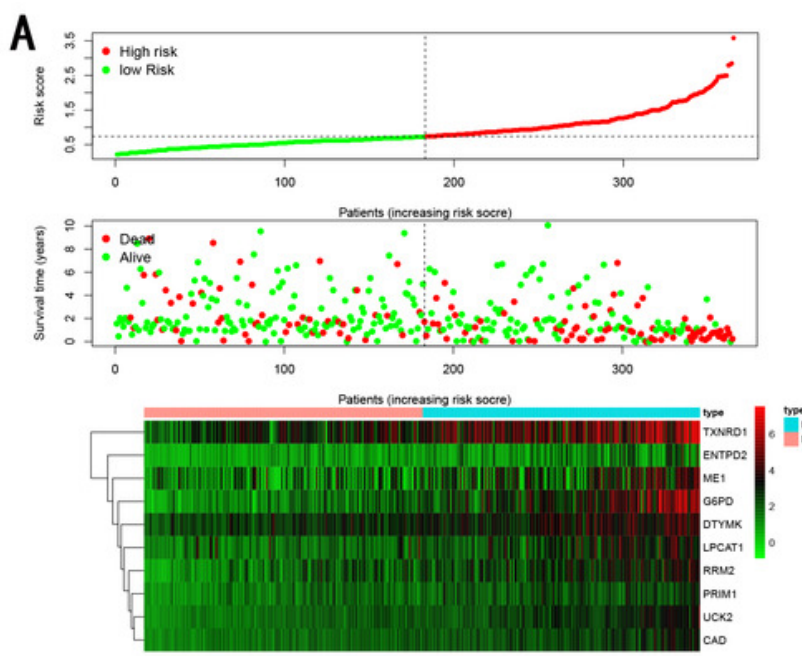

D

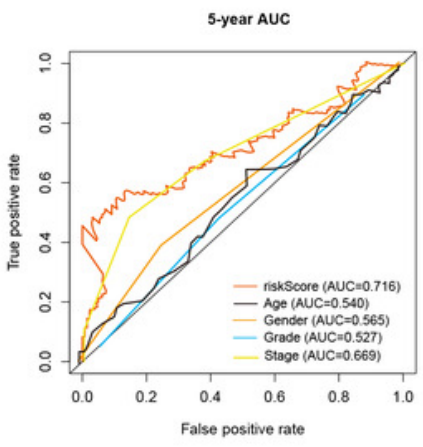

F

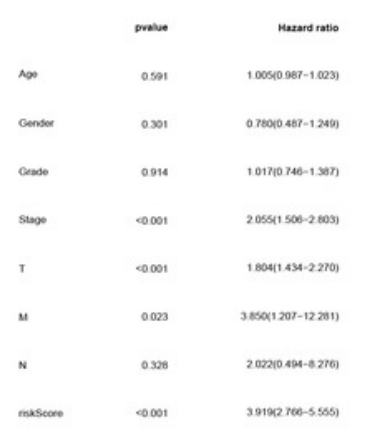

B
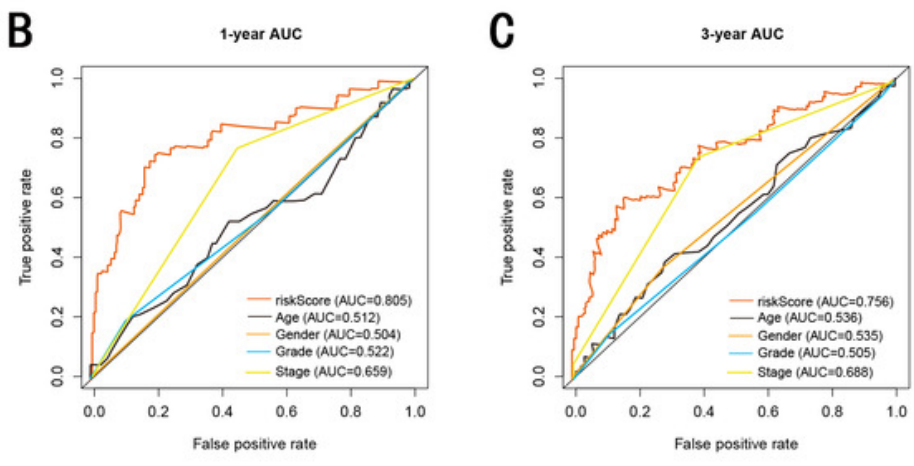

Risk + High risk $\neq$ Low risk

E

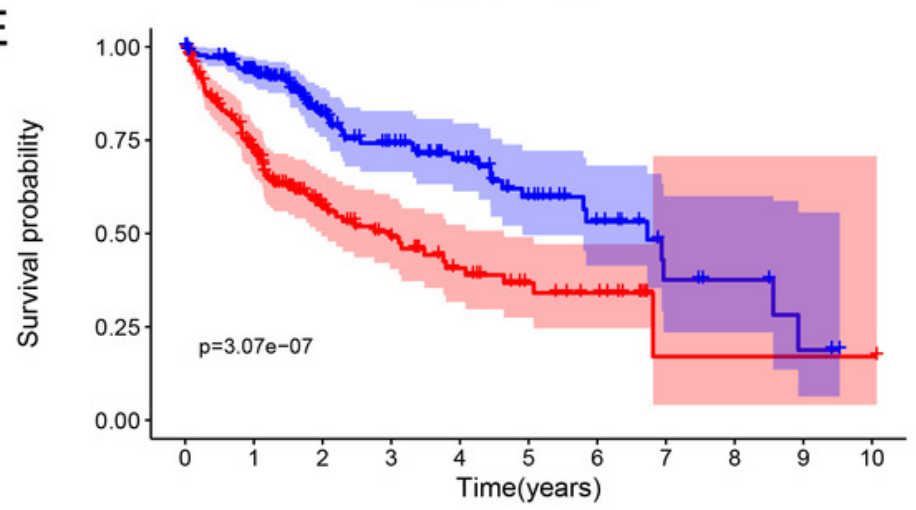

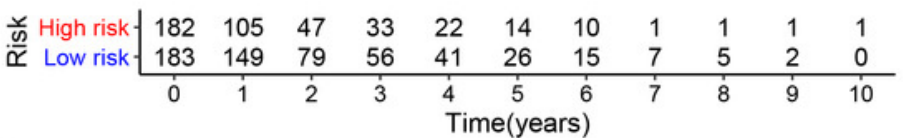

G
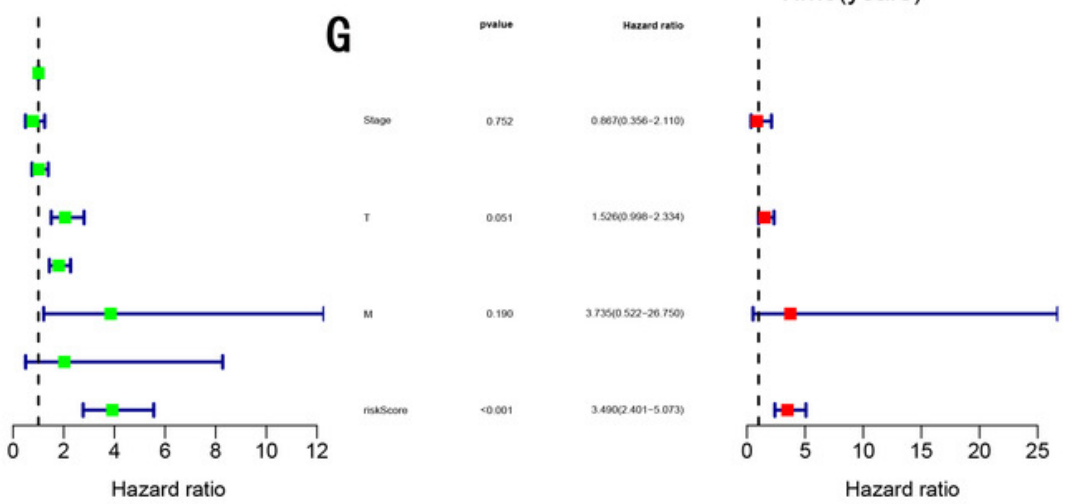


\section{Figure 5}

Gene signature performance analysis using ICGC testing cohort

(A) Distribution of 10-gene-based risk scores, patient survival durations, gene expression levels (B) 1-year ROC curve analyses of gene signature and clinical parameters (C) 3-year ROC curve analyses of gene signature and clinical parameters (D) 5-year ROC curve analyses of gene signature and clinical parameters (E) Kaplan-Meier curves of OS based on gene signature (F) Prognostic value detection of the gene signature via univariate survival-related analysis (G) Prognostic value detection of the gene signature via multivariate survival-related analysis 

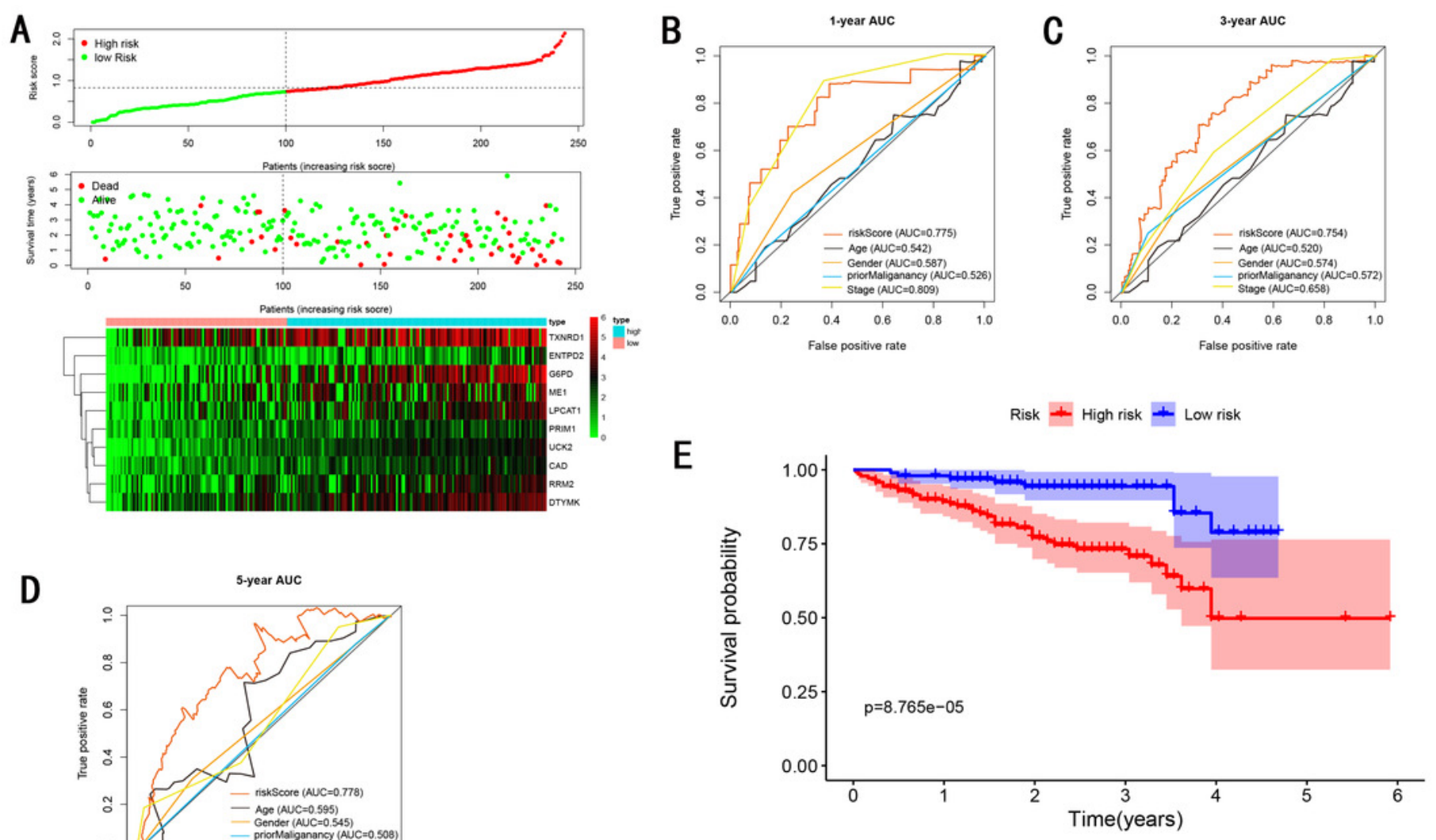

D
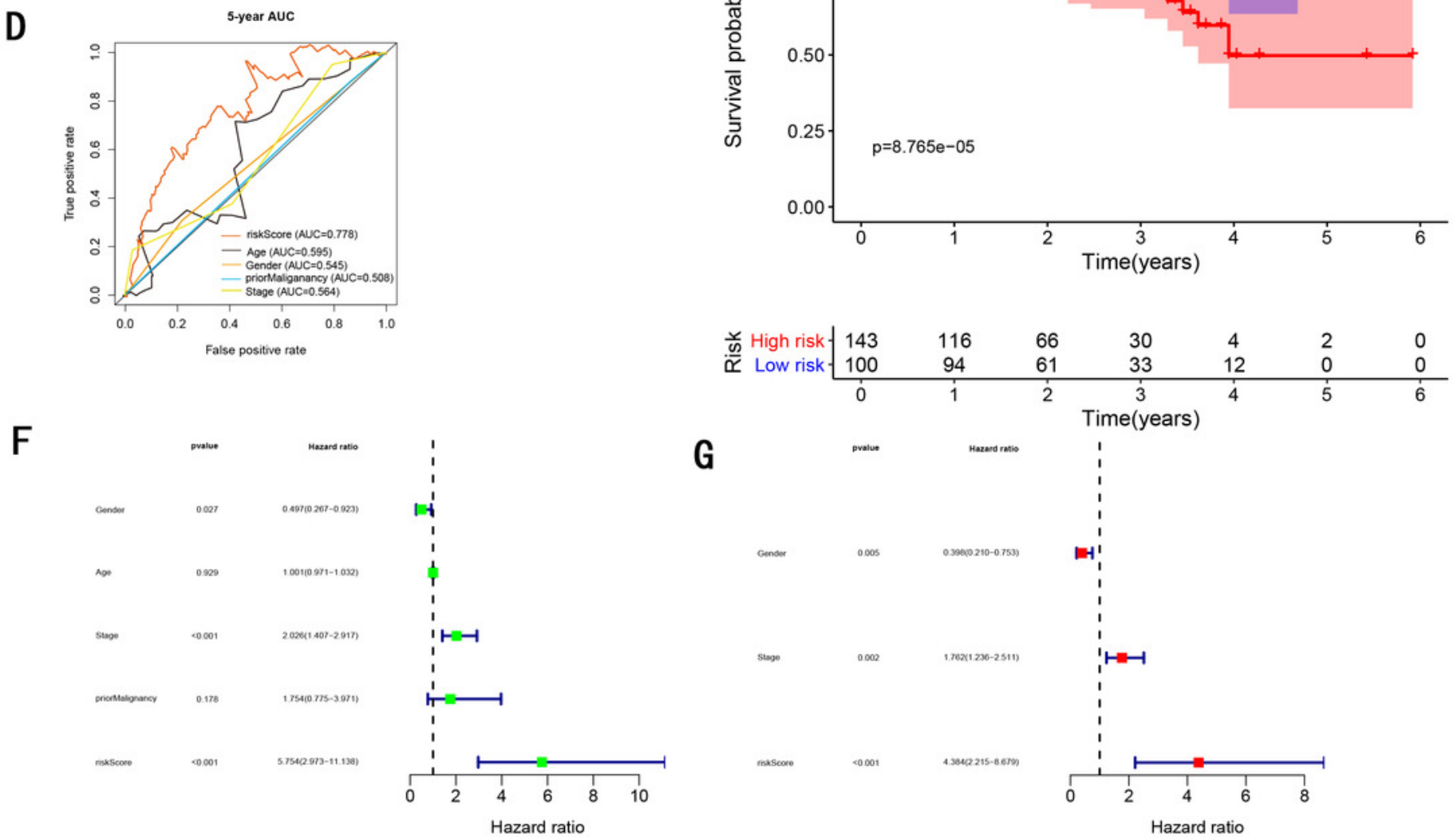

G

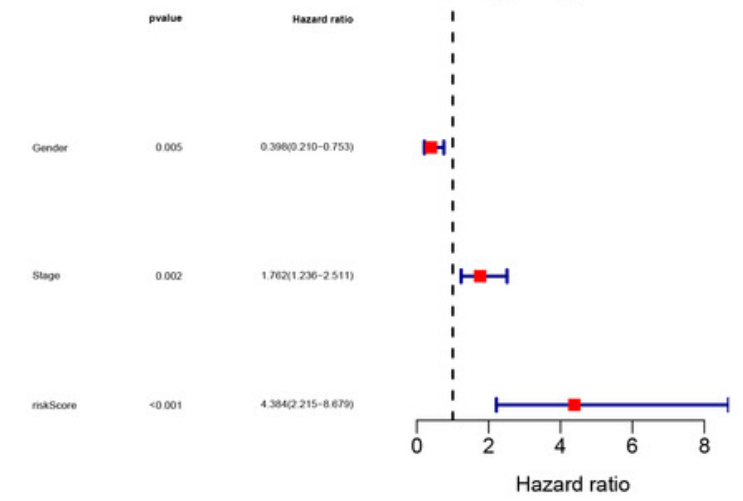




\section{Figure 6}

Gene signature performance analysis using GSE14520 testing cohort

(A) Distribution of 10-gene-based risk scores, patient survival durations, gene expression

levels (B) 1-year ROC curve analyses of gene signature and clinical parameters (C) 3-year ROC curve analyses of gene signature and clinical parameters (D) 5-year ROC curve analyses of gene signature and clinical parameters (E) Kaplan-Meier curves of OS based on gene signature (F) Prognostic value detection of the gene signature via univariate survival-related analysis (G) Prognostic value detection of the gene signature via multivariate survival-related analysis 

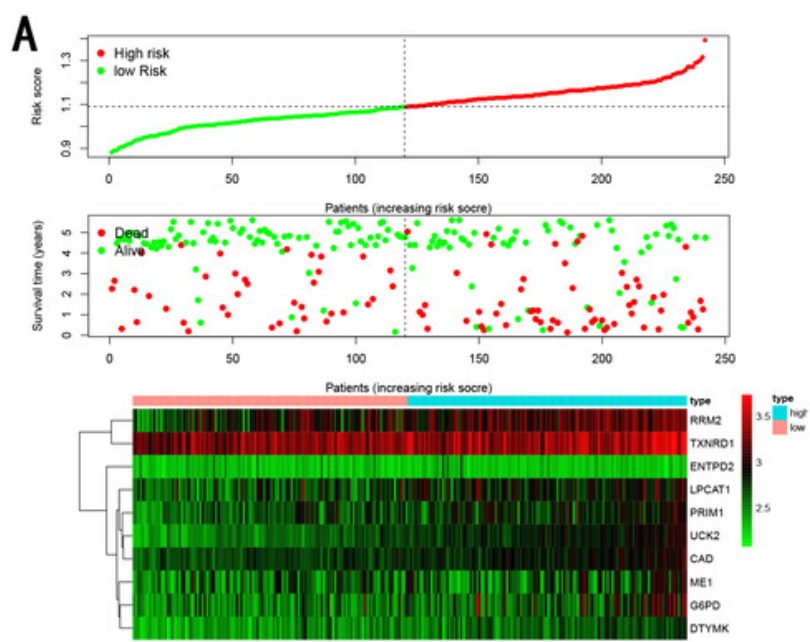

D

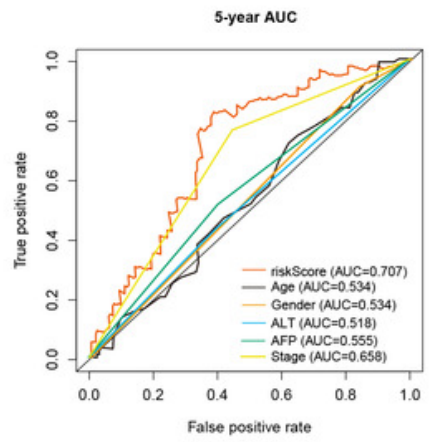

$\mathrm{F}$
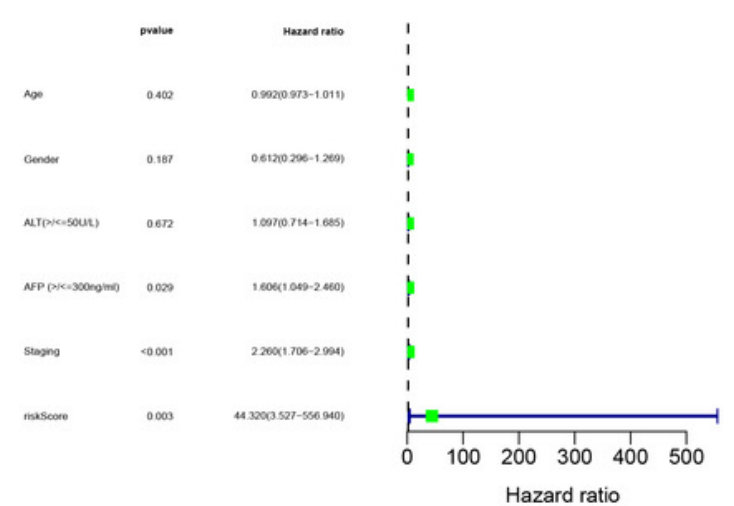

B

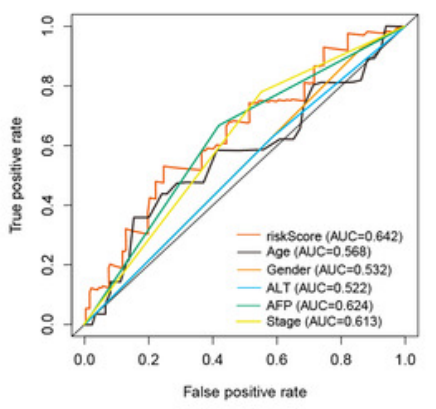

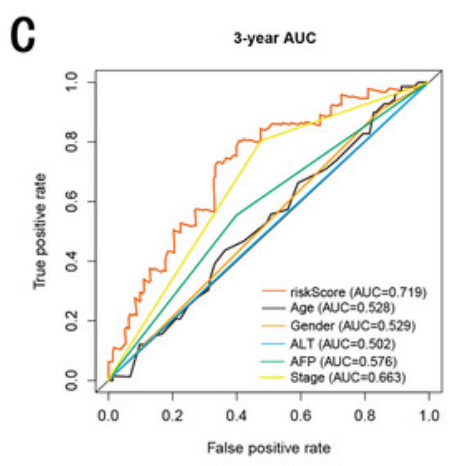

Risk + High risk + Low risk

E

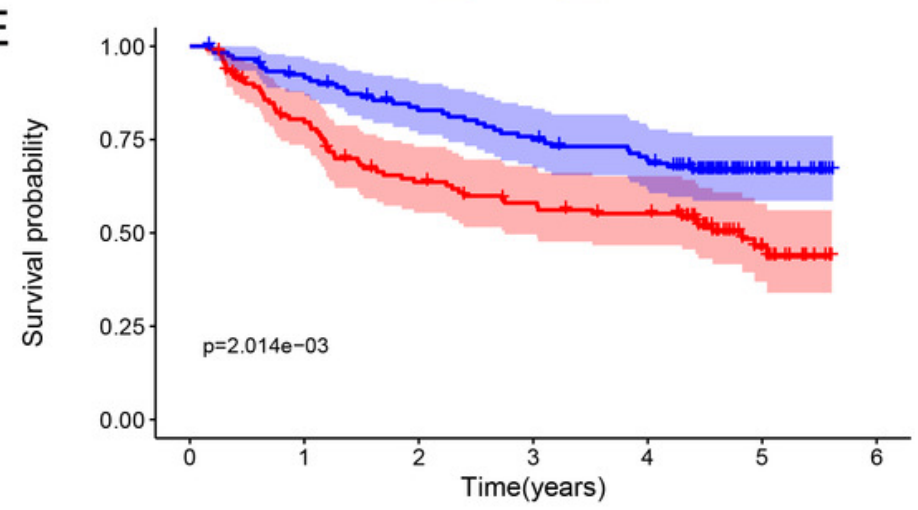

\begin{tabular}{|c|c|c|c|c|c|c|c|}
\hline $\begin{array}{l}\text { N High risk } \\
\underline{\underline{Y}} \text { Low risk }\end{array}$ & $\begin{array}{l}122 \\
120\end{array}$ & $\begin{array}{c}92 \\
108\end{array}$ & $\begin{array}{l}70 \\
94\end{array}$ & $\begin{array}{l}61 \\
85\end{array}$ & $\begin{array}{l}56 \\
77\end{array}$ & $\begin{array}{l}19 \\
24\end{array}$ & $\begin{array}{l}0 \\
0\end{array}$ \\
\hline & 0 & 1 & 2 & $\begin{array}{l}3 \\
e(y e\end{array}$ & 4 & 5 & 6 \\
\hline
\end{tabular}

G

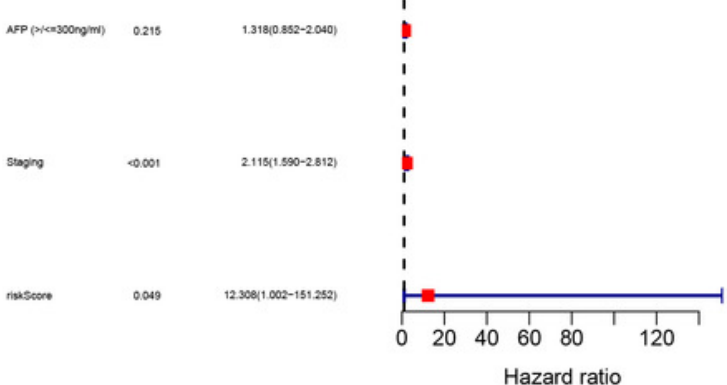




\section{Figure 7}

Gene signature performance analysis using internal testing cohort

(A) Distribution of 10-gene-based risk scores, patient survival durations, gene expression levels (B) 1-year ROC curve analyses of gene signature and clinical parameters (C) 3-year ROC curve analyses of gene signature and clinical parameters (D) 5-year ROC curve analyses of gene signature and clinical parameters (E) Kaplan-Meier curves of OS based on gene signature (F) Prognostic value detection of the gene signature via univariate survival-related analysis (G) Prognostic value detection of the gene signature via multivariate survival-related analysis 
A
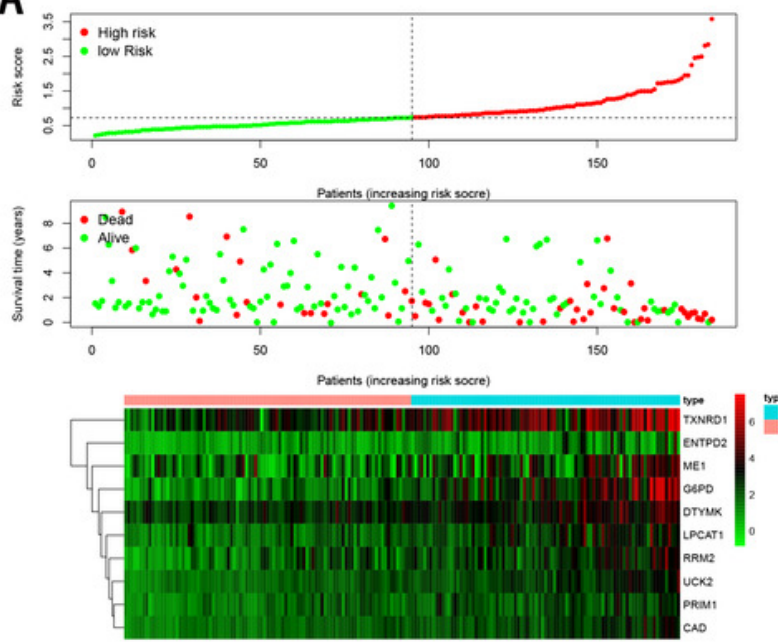

D

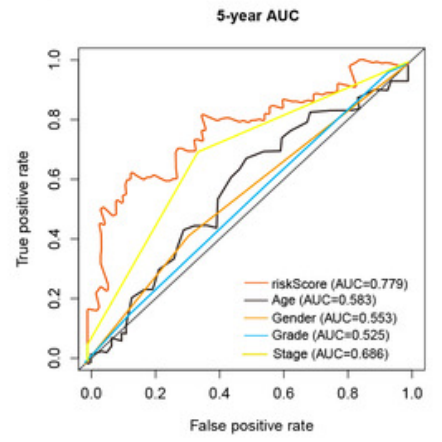

F

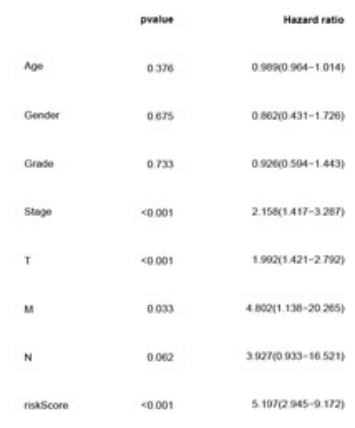

B

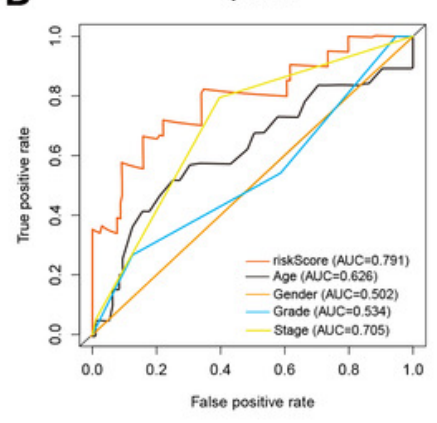

C

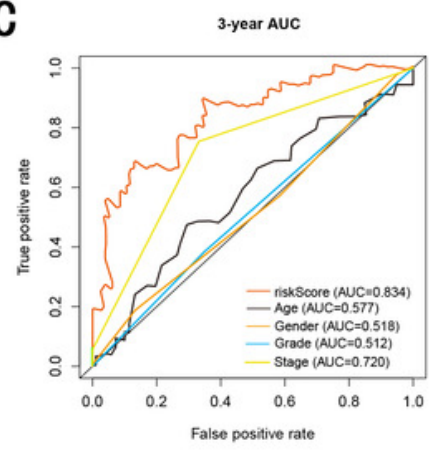

Risk + High risk + Low risk

E

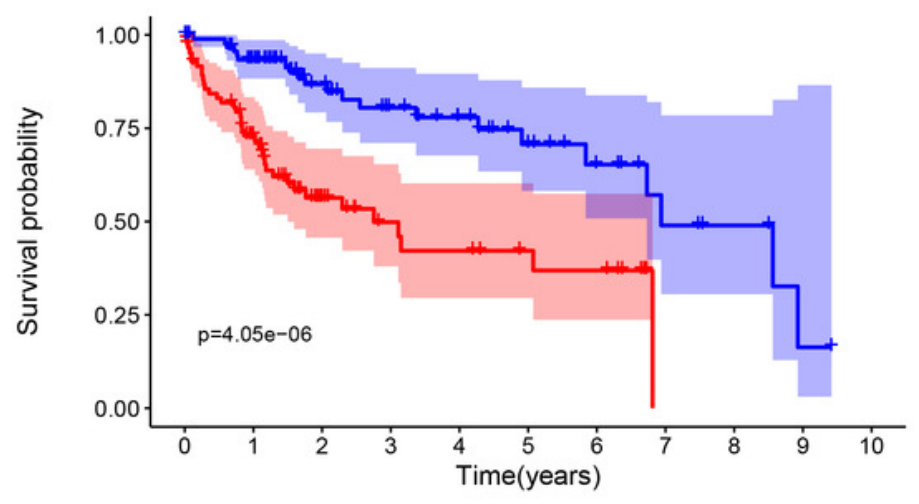

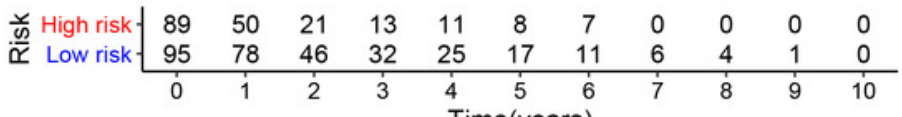

G

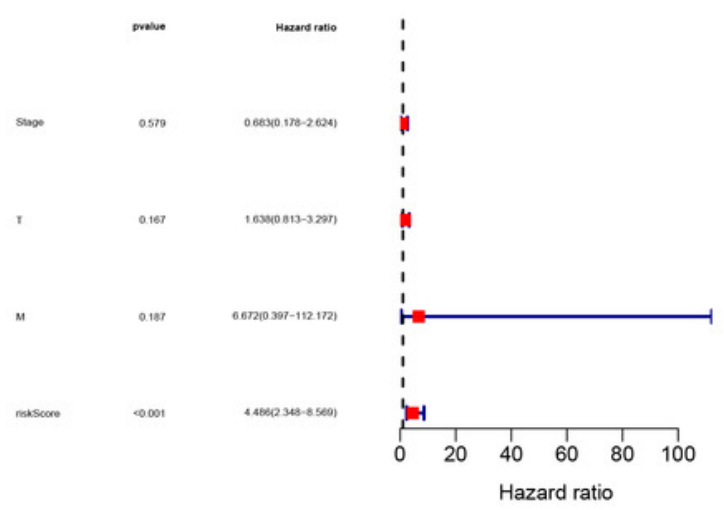




\section{Figure 8}

Gene signature performance analysis using entire testing cohort

(A) Distribution of 10-gene-based risk scores, patient survival durations, gene expression levels (B) 1-year ROC curve analyses of gene signature and clinical parameters (C) 3-year ROC curve analyses of gene signature and clinical parameters (D) 5-year ROC curve analyses of gene signature and clinical parameters (E) Kaplan-Meier curves of OS based on gene signature (F) Prognostic value detection of the gene signature via univariate survival-related analysis (G) Prognostic value detection of the gene signature via multivariate survival-related analysis 
A
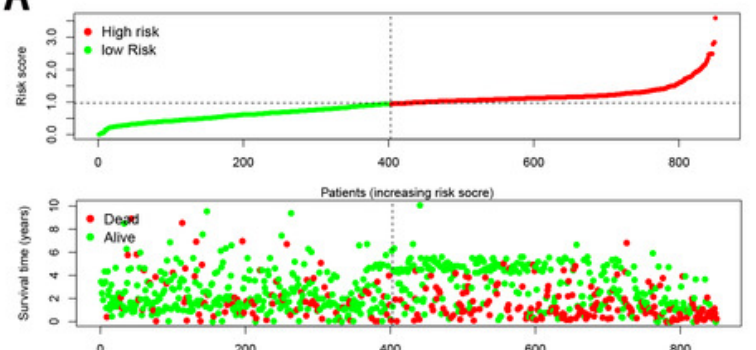

200

400

$600 \quad 800$

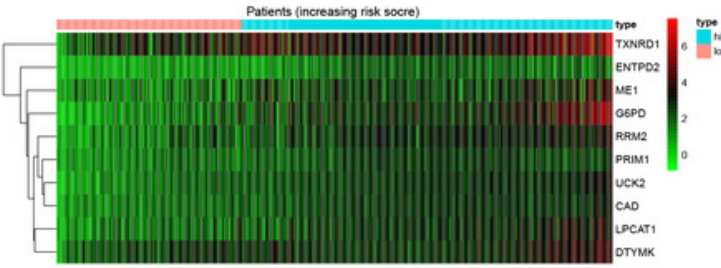

D

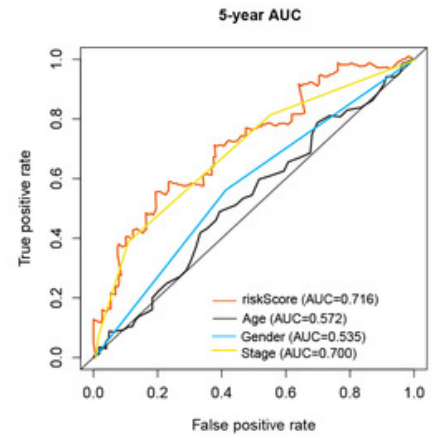

B

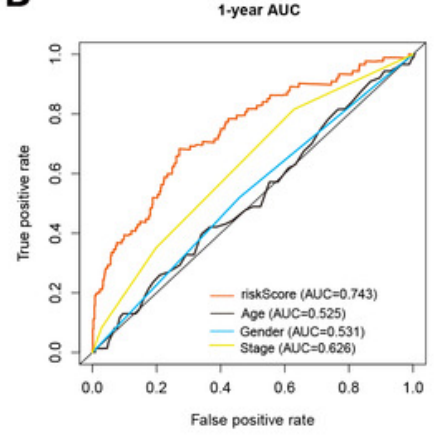

C

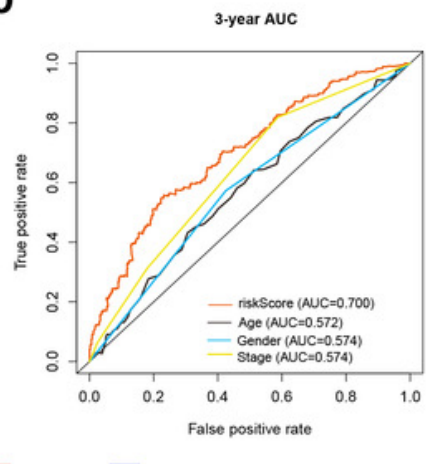

Risk + High risk + Low risk

E

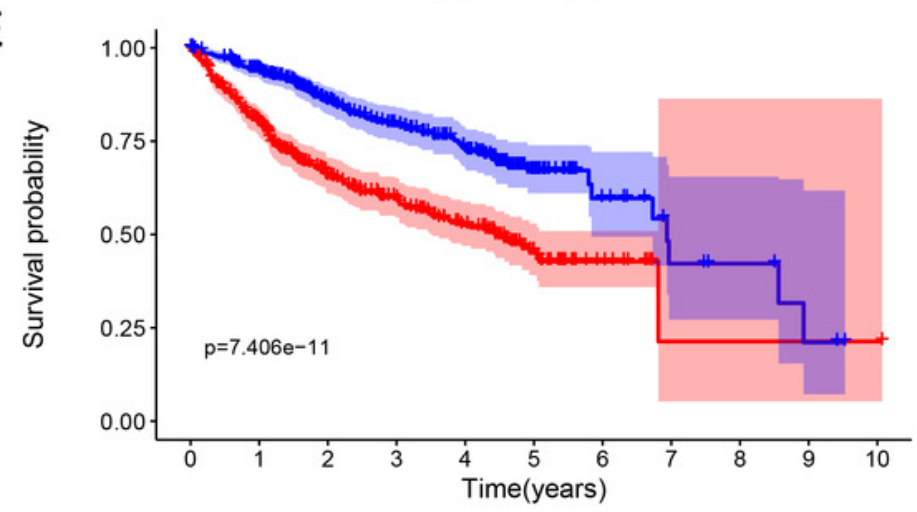

F

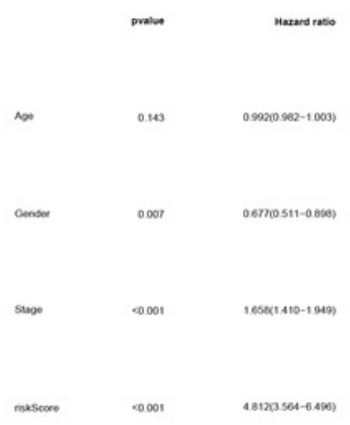

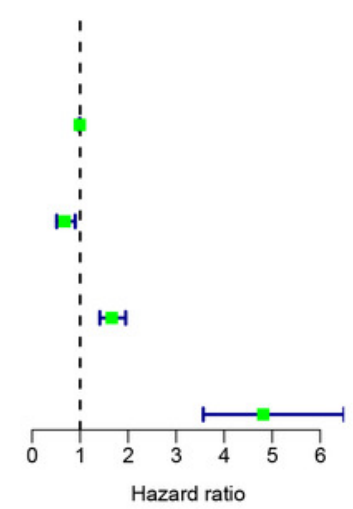

G
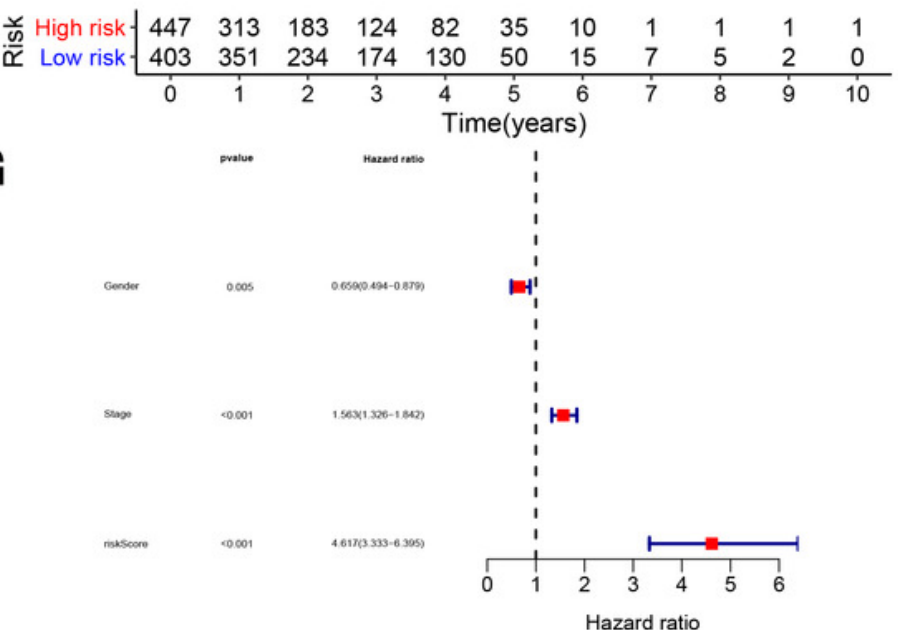
Figure 9

The predictive performance of the gene signature on OS in different subgroups stratified by clinical parameters

(A) Training cohort (B) Internal testing cohort (C) ICGC testing cohort (D) GSE14520 testing cohort (E) Entire testing cohort

A

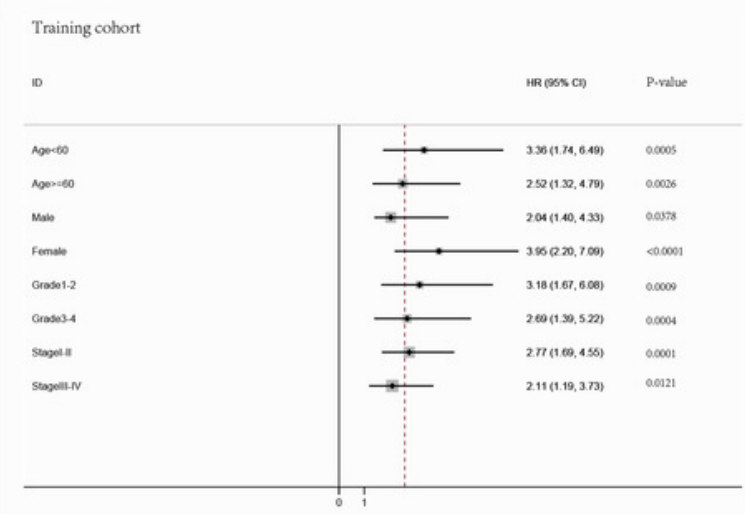

C

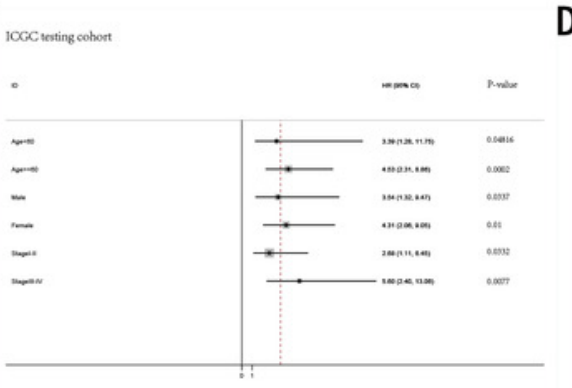

B

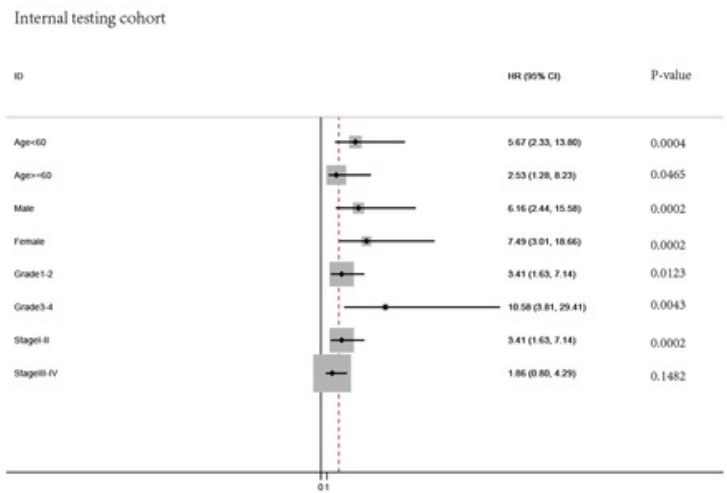

D

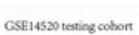

$\mathrm{E}$

\begin{tabular}{|c|c|c|c|}
\hline \multicolumn{2}{|c|}{ 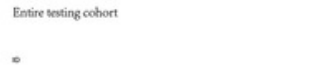 } & mema & Nom \\
\hline- & + & innerem & $=$ \\
\hline$=$ & $\leftarrow$ & 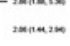 & $-\infty$ \\
\hline$=$ & 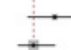 & manemer & $=$ \\
\hline$=$ & ? & тия & $\ldots$ \\
\hline
\end{tabular}

